\title{
Improving Energy Efficiency in Idle Listening of IEEE 802.11 WLANs
}

\author{
Muhammad Adnan and Eun-Chan Park \\ Department of Information and Communication Engineering, Dongguk University, Seoul 100-715, Republic of Korea
}

Correspondence should be addressed to Eun-Chan Park; ecpark@dongguk.edu

Received 21 December 2015; Revised 3 May 2016; Accepted 15 May 2016

Academic Editor: Yuh-Shyan Chen

Copyright ( 2016 M. Adnan and E.-C. Park. This is an open access article distributed under the Creative Commons Attribution License, which permits unrestricted use, distribution, and reproduction in any medium, provided the original work is properly cited.

\begin{abstract}
This paper aims to improve energy efficiency of IEEE 802.11 wireless local area networks (WLANs) by effectively dealing with idle listening (IL), which is required for channel sensing and is unavoidable in a contention-based channel access mechanism. Firstly, we show that IL is a dominant source of energy drain in WLANs and it cannot be effectively alleviated by the power saving mechanism proposed in the IEEE 802.11 standard. To solve this problem, we propose an energy-efficient mechanism that combines three schemes in a systematic way: downclocking, frame aggregation, and contention window adjustment. The downclocking scheme lets a station remain in a semisleep state when overhearing frames destined to neighbor stations, whereby the station consumes the minimal energy without impairing channel access capability. As well as decreasing the channel access overhead, the frame aggregation scheme prolongs the period of semisleep time. Moreover, by controlling the size of contention window based on the number of stations, the proposed mechanism decreases unnecessary IL time due to collision and retransmission. By deriving an analysis model and performing extensive simulations, we confirm that the proposed mechanism significantly improves the energy efficiency and throughput, by up to 2.8 and 1.8 times, respectively, compared to the conventional power saving mechanisms.
\end{abstract}

\section{Introduction}

Nowadays, wireless local area networks (WLANs), also referred to as Wi-Fi, are widely deployed almost everywhere due to low installation cost and easy accessibility to the Internet. At the same time, most mobile devices (e.g., smart phones and tablets) and wearable devices (e.g., smart watches and smart glasses) are equipped with Wi-Fi interface, and it is expected that $\mathrm{Wi}$-Fi will be increasingly installed in various emerging consumer electronics and embedded systems by keeping pace with the development of the Internet of Things. However, one of the most crucial problems faced in these devices is the limited capacity of energy storage and the significant energy drain by the WLAN interface. The radio interface is a source of weighty energy drain in mobile devices [1]; the WLAN interface card consumes almost half of the total energy [2]. Thus, it is imperative to manage the energy consumption of WLAN interfaces for extending the battery lifetime of mobile devices.

In order to limit the energy consumption at the radio interface, the power saving mode (PSM) was introduced in the IEEE 802.11 standard [3]. In PSM, a mobile station (STA) periodically switches between sleep and active states, where the radio interface is turned off during the sleep state. The energy consumption of an STA deploying PSM can be reduced by controlling the period or frequency of active and sleep states. However, the PSM is effective only in the case where STAs occasionally have frames to receive, and it does not make any significant contribution to energy conservation in the active state. It was shown in [4] that if STAs have a moderate amount of traffic to send/receive, more than 80 percent of the time is spent in idle listening (IL), even though the PSM is deployed. According to the channel access mechanism of WLANs, that is, carrier sense multiple access (CSMA), there are three states of STAs: frame transmission, frame reception, and IL. In the IL state, an STA performs channel sensing to determine whether the channel is idle or busy. Before transmitting a frame, the STA should defer the transmission during a random backoff time. This procedure of channel sensing and backoff is inevitable in the contentionbased channel access mechanism. Moreover, the STA spends its time and energy when it receives frames that are not 
destined to itself, which comprises another part of IL. Let us denote an intended (nonintended) frame as a frame whose destination address is equal to (different from) the address of the STA listening to the channel. Due to the nature of carrier sensing and broadcasting in WLANs, the energy consumption in overhearing a nonintended frame is comparable to that in receiving an intended frame. Hence, the amount of energy consumption in the state of IL can be rather higher than those in the states of transmission and/or reception, especially when the number of STAs is large or their traffic loads are heavy. Meanwhile, a failure of frame transmission due to collision leads to the increase of IL time (ILT) in the STA suffering from the failure, as well as in those of the other STAs sensing the channel. In addition to the energy wastage due to retransmission, a binary exponential backoff (BEB) algorithm invoked by the transmission failure doubles the size of the contention window and accordingly increases the ILT due to backoff. As a result, the ILT is unavoidable due to the nature of the channel access mechanism of WLAN, and it is essential to effectively deal with IL for improving device's energy efficiency.

This paper aims to conserve the IL-related energy in WLANs. For this purpose, we consider two approaches: reducing the unnecessary energy consumption during ILT and decreasing ILT. To realize these two approaches simultaneously, we propose a combination of three schemes: (i) downclocking, (ii) frame aggregation, and (iii) contention window control. With the downclocking scheme, the clock frequency of the WLAN interface card is lowered from the full operation clock frequency when receiving nonintended frames (we refer to this state as semisleep), similar to [4, 5]. Once the receiving frame is identified as a nonintended frame, the STA switches to the semisleep state until the end of transmission time of the nonintended frame. The gain of downclocking is amplified by introducing the frame aggregation scheme, which is the second component of the proposed mechanism. Since the frame aggregation scheme allows the STA to transmit multiple frames per channel access, it prolongs the semisleep time in the neighbor STAs to conserve more energy by means of downclocking. In addition to improving the energy efficiency, the frame aggregation is effective to improve the transmission efficiency by decreasing channel access overhead per frame, for example, backoff time and header overhead. The third component, contention window control, is designed to deal with the ILT resulting from collision, which becomes severe as the number of STAs competing for the channel access increases. By scaling the size of contention window depending on the number of STAs, the proposed mechanism can mitigate collision, and thus, it can prevent the unnecessary energy consumption due to collision and retransmission. We adopt the basic idea of frame aggregation and contention window control in our previous work [6].

Via rigorous analysis and extensive simulations, it is shown that, compared to the conventional PSM, the proposed mechanism reduces the energy per bit by up to about 2.8 times, while maintaining up to about $80 \%$ higher throughput. The following points summarize the notable features of the proposed mechanism and the contributions of this study. (i) The proposed mechanism combines three schemes (downclocking, frame aggregation, and contention window control) in a systematic way without requiring critical changes in the current IEEE 802.11 standard; therefore, it can be a feasible and practical solution for diminishing the unnecessary energy consumption due to IL in WLANs.

(ii) As well as improving energy efficiency, the proposed mechanism increases transmission efficiency by employing frame aggregation and contention window control, which decrease the channel access overhead and collision, respectively.

(iii) An effective analysis model is derived to evaluate the effectiveness of each component of the proposed mechanism in terms of energy per bit and throughput. In addition, the analysis result confirms that the downclocking scheme alone cannot significantly decrease the energy consumption due to IL and that the effect of downclocking can be quite magnified when it is integrated with the schemes of frame aggregation and contention window control.

The rest of this paper is organized as follows. Section 2 gives the background and motivation of this study and addresses the related work. Section 3 provides details of the proposed mechanism, and its analytical model is derived in Section 4. Section 5 presents the simulation results of the proposed mechanism under various conditions. The conclusion follows in Section 6.

\section{Motivation and Related Work}

2.1. Background of PSM. We consider the PSM in an infrastructure WLAN where STAs transmit/receive frames to/from an access point (AP). According to the PSM, STAs alternate active (or awake) state and sleep (or doze) state, and the AP buffers frames for STAs in the sleep state. AP broadcasts a traffic indication map (TIM) periodically to inform the STAs of the existence of pending frames destined to them, while STAs wake up periodically to listen for the TIM. The TIM is embedded in a beacon frame, and the time difference between two consecutive beacon frame transmissions is called as beacon interval (BI). In the active state, the STA can trans$\mathrm{mit} /$ receive its frames or sense the channel during backoff and reception of nonintended frames, which accounts for the ILT. The energy consumption of STA in the active state depends upon several factors like traffic load, frame size, transmission rate, and the length of ILT. On the contrary, in the sleep state, the PSM-enabled STA turns its radio interface off and is not capable of transmitting/receiving any frames; thus, the energy consumption in the sleep state can be significantly reduced. In addition to the BI, there is another interval, listen interval (LI), which affects the period and length of active and sleep states. The LI indicates the period at which a PSMenabled STA wakes up to listen for the TIM. According to the IEEE 802.11 PSM, the LI should be set to multiple of BI. For example, if the LI is set to $3 \mathrm{BIs}$, the STA listens to the TIM only at every third beacon frame and ignores the other two TIMs. The value of LI is determined by the negotiation 
between the STA and AP in the procedure of association. If an STA hears the TIM indicating pending frames, it should be in the active state until receiving the pending frames. In order to retrieve the pending frames, the STA initiates a PS-Poll message to the AP after a random backoff time. Once the AP receives the PS-Poll message from the STA, it transmits the data frame to the corresponding STA after a short interframe space (SIFS). One PS-Poll message needs to be transmitted to retrieve one data frame from the AP, followed by an acknowledgment (ACK) from the STA. If there are multiple pending frames that need to be delivered to the STA, the AP sets "More data bit" field in the data frame. Then, the STA initiates another PS-Poll message to receive its additional frame from the AP. After receiving all the pending frames, the STA switches to the sleep state to conserve energy. If the STA hears the TIM indicating no pending frame, it can sleep immediately after receiving the TIM. It is important to note that the PS-Poll message is transmitted in a contention-basis manner; that is, the PS-Poll message cannot be transmitted as soon as the STA recognizes the existence of pending frames by receiving the TIM, but it can be transmitted after competing with other STAs (or APs) transmitting data frames or PS-Poll messages. Therefore, this contention incurs an unavoidable delay in transmitting the PS-Poll message, which increases the ILT and degrades the energy efficiency in the active state.

2.2. Motivation. The PSM can save energy by allowing STAs to remain in the sleep state, as explained in Section 2.1, and there have been lots of studies in the literature to optimize the performance of PSM by tuning parameters like BI and LI and by enhancing buffer management in the AP $[7,8]$. However, the PSM cannot make any contribution to energy efficiency when STAs in the active state retrieve the pending frames and it cannot effectively deal with the ILT due to the nature of contention-based channel access of IEEE 802.11 WLANs, which becomes severer as the number STAs increases.

In order to quantify the amount of energy spent due to the ILT, we perform a simple simulation. We consider that one PSM-enabled STA coexists with several background (nonPSM-enabled) STAs, whose number (denoted as $N_{\mathrm{bg}}$ ) varies from 2 to 20 . We set BI and LI to $100 \mathrm{~ms}$ and 2 BIs, respectively. In the simulation, we assume that the background STAs always have data frames to transmit, while the PSM-enabled STA always has data frames to retrieve in the first BI within the LI, but it has no frame to retrieve in the other BI within the LI (with this artificial configuration, we can make a balance between active time and sleep time in the PSM-enabled STA. In Section 5, we will investigate the effect of traffic load via various simulations). Details about simulation configuration can be found in Section 5. Note that the PSM-enabled STA has three states: IL, sleep, and active (transmission and reception) and that the power consumed due to transmission is comparable to that due to reception $[2,4,9]$. Figure 1 compares the total energies consumed in these three states, denoted as $\epsilon_{\mathrm{IL}}, \epsilon_{\mathrm{SL}}$, and $\epsilon_{\mathrm{AC}}$, respectively. From Figure 1, we can observe that the STA, even with the PSM, consumes most of its energy due to IL; for example, when $N_{\mathrm{bg}}=10, \epsilon_{\mathrm{IL}}$ exceeds $7.1 \mathrm{~J}$, which is higher than $\epsilon_{\mathrm{AC}}$ by more than 6 times and corresponds to about $80 \%$ of total energy consumption.

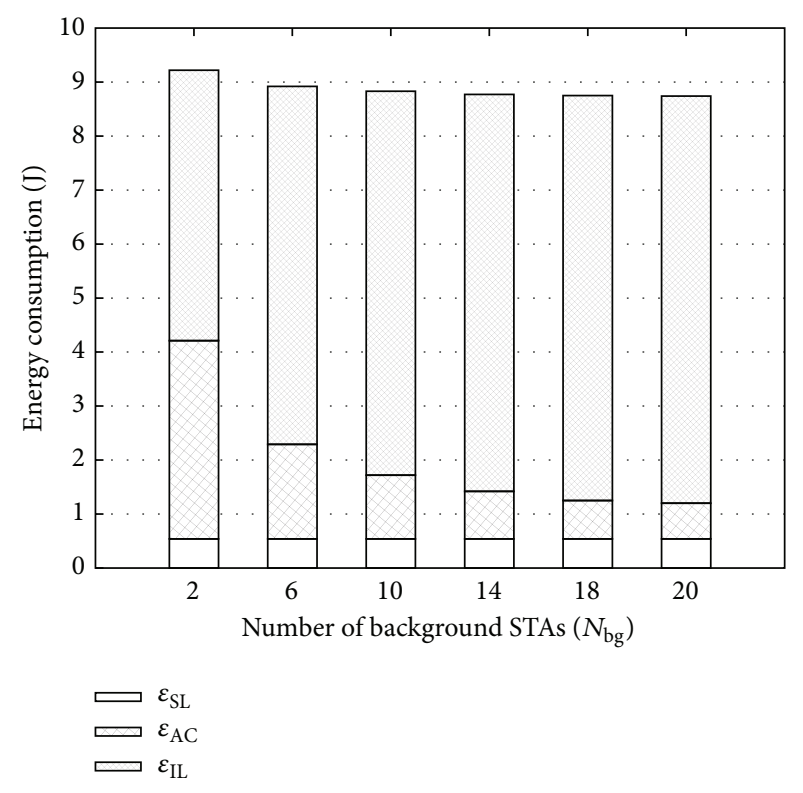

FIGURE 1: Comparison of energy consumption in idle listening, sleep, and active (transmission and reception) states.

In addition, as $N_{\mathrm{bg}}$ increases, $\epsilon_{\mathrm{IL}}$ increases but $\epsilon_{\mathrm{AC}}$ decreases; that is, the ILT increases and energy efficiency degrades with respect to the increase in the number of competing STAs. On the other hand, $\epsilon_{\mathrm{SL}}$ is almost constant, regardless of $N_{\text {bg }}$, because $N_{\text {bg }}$ hardly affects the sleep time under this simulation configuration.

2.3. Related Work. Firstly, we address features of several power saving mechanisms adopted in the IEEE 802.11 standards. Then, we focus on studies dealing with the IL energy.

To improve energy efficiency and quality of service (QoS), an enhanced power saving mechanism, Unscheduled Automatic Power Save Delivery (U-APSD), was introduced in the standard of IEEE 802.11e [10]. Unlike PSM, an STA under U-APSD does not have to wake up periodically to listen for the TIM, but it transmits a trigger frame (QoS data frame or null frame) at any time to retrieve the pending frames in the AP buffer. On receiving the trigger frame from the STA, the AP firstly acknowledges it, and then if the AP has pending frames, the AP performs the channel access procedure and transmits several pending frames to the STA successively within the transmission opportunity (TXOP) limit. After receiving all the frames from the AP, the STA can go back to sleep. Since several frames can be retrieved by a single trigger in U-APSD, the energy efficiency can be improved compared to the PSM. In determining the frequency of trigger frame transmission, there occurs a trade-off; the frequent triggering can reduce the retrieval delay of pending data frame, but it may increase collision and waste airtime or energy due to unnecessarily frequent trigger frames $[9,11]$.

Recently, IEEE 802.11n introduced a scheduling-based power saving mechanism, power save multipoll (PSMP) [12]. This mechanism allows an AP to schedule downlink and uplink transmissions for multiple STAs by broadcasting 
a single PSMP frame. On receiving the PSMP frame, each STA becomes aware of when to wake up to receive/transmit data frames. Thanks to the scheduling-based operation, the PSMP mechanism can improve the energy efficiency by avoiding the ILT spent in sensing the channel and receiving nonintended frames, and at the same time, it relieves the overhead of individual polling or triggering by utilizing the PSMP frame. However, the PSMP mechanism has a few drawbacks. The overhead of PSMP management frames and the implementation complexity increase as the number of STAs increases. Moreover, if the PSMP frame is corrupted or misinterpreted due to any reason (e.g., wireless channel error or interference), the scheduled airtime may be wasted or some STAs may remain awake throughout the whole PSMP duration $[13,14]$.

Moreover, many mechanisms have been proposed to reduce the energy dissipation in the ILT. Considering that the energy is the product of power and time, they can be classified into two categories: (i) decreasing power in the ILT $[4,15-$ 17] and (ii) decreasing the ILT [18-22]. The first approach makes STAs switch to a low-power state (e.g., semisleep) in the ILT, without attempting to decrease the ILT, whereas the second approach aims to minimize ILT resulting from channel access, collision, or reception of nonintended frames.

The mechanism proposed in [15] employs the ready-tosend/clear-to-send (RTS/CTS) control messages to limit the energy expenditure due to overhearing nonintended frames. If an STA overhears an RTS or CTS frame that is not destined to it, the STA forces its radio interface to a low-power idling state for the duration specified in the RTS or CTS frame. In addition, the mechanisms proposed in $[4,16]$ adopt a similar approach to that of [15]. The mechanism in [4] proposes a new preamble where the destination address is embedded and proposes a novel detection technique so that the STAs can accurately detect nonintended frames even with the reduced clock frequency. Similarly, the mechanism in [16] detects nonintended frames based on the information of MAC header and proposes adding a checksum field in the MAC header to decrease the probability of false detection. The energy-efficient distributed access mechanism proposed in [17] aims to reduce energy consumption during the backoff procedure, as while overhearing nonintended frames by forcing the STA to remain in a low-power state during the backoff procedure.

The energy efficiency can also be improved by decreasing ILT. The micropower management mechanism proposed in [18] lets STAs sleep for a very short idle time (in the order of microseconds) by leveraging a prediction mechanism of frame arrival, so that the short ILT is transformed to sleep time. Similar to PSMP, the studies in [19-22] propose a scheduling-based or reservation-based approach to avoid unnecessary ILT. The mechanism in [19] proposes dividing the frame into a control subframe for channel contention and data subframe for data transmission, so that it can be free from unnecessary energy dissipation for accessing the channel and for receiving nonintended or collided frames. In the approaches of $[20,21]$, the time is slotted and reserved after exchanging control frames between transmitter and receiver [20], or the AP schedules the transmission order/duration and assigns the slotted time to a specific STA [21]. In this way, only STAs involved in communication wake up during the designated time, and other STAs can go to sleep. The mechanism in [22] proposes a prioritized reservation scheme, with which the contention among PS-Poll messages can be avoided and STAs are allowed to retrieve data frames at the reserved time.

Compared to these previous approaches, our mechanism has several advantages and desirable properties as follows.

(i) The approach of decreasing power in the ILT [4, 1517] has a limited effect on energy efficiency, especially when the transmission rate is high or frame size is small. We validate the weakness of this approach via analysis and simulation and propose an effective and simple solution to magnify the benefit of this approach.

(ii) The approach based on scheduling or reservation [1922] requires significant modifications of the channel access mechanism in the current IEEE 802.11 WLANs, such that it cannot maintain a backward compatibility. Our mechanism can be practically implemented without violating the compliance with the IEEE 802.11 standard.

(iii) None of the previously discussed approaches $[4,10,12$, 15-22] deal with collision and retransmission, which is another primary source of energy dissipation and performance degradation. Our mechanism incorporates an effective solution to collision for energy efficiency and performance enhancement.

\section{Proposed Mechanism}

In order to conserve energy consumption due to IL, the proposed mechanism systematically combines three schemes: (i) downclocking, (ii) frame aggregation, and (iii) contention window control. The first scheme, downclocking, lowers the clock frequency of radio interface during the ILT. When detecting nonintended frames, the STA switches to the semisleep state by decreasing the clock frequency without impairing the function of carrier sensing. The second scheme, frame aggregation, is designed to boost the gain of downclocking by prolonging the time of semisleep. The frame aggregation mechanism lets STAs transmit multiple frames per channel access; thus, it extends the semisleep time of neighbor STAs overhearing nonintended frames and decreases the ILT required for channel sensing in the transmitting STAs. Lastly, the third scheme, contention window control, contributes to the decrease of collision among STAs. Therefore, it can avoid unnecessary energy waste due to collision and retransmission. In this way, the proposed mechanism not only reduces the power in the ILT but also decreases the length of ILT. Moreover, the proposed mechanism does not require any significant changes in the current standard of IEEE 802.11. The following subsections explain the details of each scheme in turn.

3.1. Downclocking in Idle Listening Time. The objective of downclocking is to curtail unnecessary expenditure of energy 
during the ILT. To explain the basic concept of downclocking, the circuitry of the radio interface needs to be considered. The primary source of energy consumption in the wireless interface card is the clock used for sampling of the incoming signal. The power consumption of the wireless interface card, $P$, follows $P \propto V^{2} f$ [23], where $V$ is the supplied voltage and $f$ is the frequency of clock. Based on this equation, there is a linear relationship between $P$ and $f$. However, in a practical system, the actual power is not exactly proportional to the frequency, because of the power consumed by other parts of the device. In $[4,5]$, the idea of reducing the clock frequency of processor in the wireless interface card was introduced to decrease the energy consumption during the ILT. The authors in [4] validated this idea via real experiments; the energy consumption during ILT is reduced to almost half when the clock frequency is reduced to $1 / 4$ of the original clock frequency. In addition, it was shown in [4] that the switching time between full clock frequency and 1/4 clock frequency is about 130-150 microseconds. These results support the technical ground for energy conservation in the ILT by means of downclocking.

In this work, we adopt the downclocking technique to reduce energy consumption in the STA overhearing nonintended frames. To perform the operation of downclocking, the STA has two requirements: (i) distinguishing nonintended frames from intended frames and (ii) obtaining the information on the transmission duration of nonintended frames. Whenever receiving (or overhearing) an incoming frame, whether intended or not, an STA reads the PHY header of the frame. The transmission duration can be calculated from "LENGTH" and "RATE" fields in the PHY header, which represent the frame size and transmission rate, respectively. After determining the duration of the incoming frame, the STA checks whether the frame is an intended frame or nonintended frame by reading the "Address 1 " field in the MAC header, which indicates the MAC address of the receiver. Then, the STA makes a decision on downclocking. Let us denote $T_{\mathrm{rx}}$ and $T_{\text {tran }}$ as the duration of incoming frame and the transition time required for switching receiving state and semisleep state, respectively. The STA switches its state to semisleep state by lowering the clock frequency by $1 / 4$ if the following two conditions are satisfied: (i) the receiver address is neither its MAC address nor a broadcast/multicast address and (ii) $T_{\text {rx }}$ is larger than $2 T_{\text {trans }}$. Otherwise, the STA does not change its clock frequency. The semisleep state is terminated (i.e., the clock frequency is returned to the original clock frequency) earlier than the expiration time of $T_{\mathrm{rx}}$ by the time amount of $T_{\text {tran }}$. As a result, the duration of semisleep state, denoted as $T_{\text {ss }}$, becomes

$$
T_{\mathrm{ss}}=\max \left(0, T_{\mathrm{rx}}-2 T_{\text {trans }}-T_{\mathrm{h}}\right),
$$

where $T_{\mathrm{h}}$ is the time spent in reading the address field of MAC header. If the PHY or MAC header of an incoming frame is corrupted, the STA cannot correctly obtain the information required for semisleep, and it discards the incoming frame and does not switch to the semisleep state. However, the probability of $\mathrm{PHY} / \mathrm{MAC}$ header corruption is insignificant because its size is usually smaller than that of payload by three orders of magnitude. Therefore, the corruption of PHY/MAC
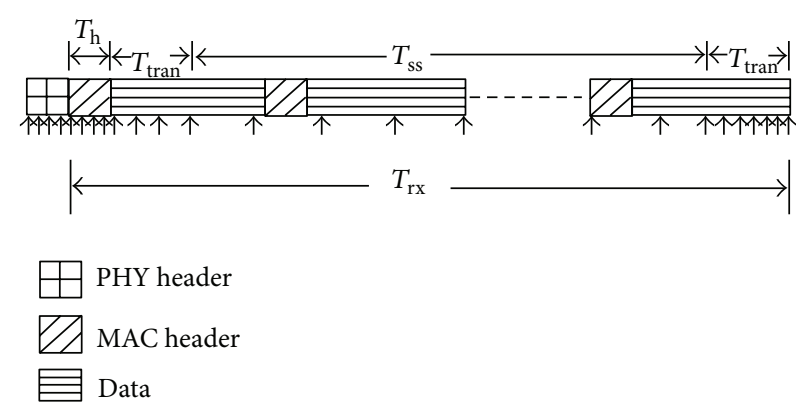

FIGURE 2: Operational example of downclocking combined with frame aggregation.

header does not make any critical adverse effect on the operation of power saving and network performance. Figure 2 shows the operation of downclocking with frame aggregation, which will be explained in the following subsection.

3.2. Frame Aggregation. We devise the frame aggregation scheme to amplify the gain of downclocking by prolonging the semisleep time. As shown in (1), the semisleep time, $T_{\mathrm{ss}}$, is determined by the receiving time of the nonintended frame, $T_{\mathrm{rx}}$, which depends on the frame size and transmission rate. Therefore, if the frame size is small or the transmission rate is high, the gain of downclocking becomes insignificant. This deficiency can be overcome by the frame aggregation. Frame aggregation in WLANs was originally introduced to enhance the channel efficiency by reducing several MAC layer overheads including backoff time, header overhead, and ACK transmission time. According to the IEEE 802.11n standard [12], there are two types of frame aggregation: aggregation of MAC service data unit (A-MSDU) and aggregation of MAC protocol data unit (A-MPDU). When aggregating multiple frames, A-MSDU causes less header overhead (i.e., a single PHY/MAC header for all the frames) than A-MPDU (i.e., a common PHY header for all the frames and individual MAC header for each frame). However, A-MPDU outperforms AMSDU in an error-prone wireless channel because the former selectively retransmits only the corrupted frames, while the latter has to unnecessarily retransmit all the frames even though only a frame is corrupted. Both schemes, as well as the combination of A-MSDU and A-MPDU or any other frame aggregation scheme like [24], can be easily integrated with the downclocking mechanism, but we consider A-MPDU in this paper due to its robustness to channel error and simple implementation.

We propose that the number of frames to be aggregated and transmitted per channel access (called as aggregation factor (AF)) should be proportional to the transmission rate of each STA. Defining the transmission rate as $R_{\mathrm{tx}}$, AF can be simply calculated as

$$
\mathrm{AF}=\min \left(\left[\alpha \frac{R_{\mathrm{tx}}}{R_{\min }}\right], N_{\mathrm{q}}\right) .
$$

Here, $\alpha$ is a positive scaling factor, $[x]$ represents the roundoff value of $x, R_{\min }$ is the minimum value of transmission rate defined in the IEEE 802.11 standard (e.g., $6 \mathrm{Mb} / \mathrm{s}$ for IEEE 
$802.11 \mathrm{a} / \mathrm{g}$ ), and $N_{\mathrm{q}}$ is the number of frames backlogged in the transmission queue when it gets the chance of channel access. The underlying rationale behind (2) is as follows:

(1) To increase the semisleep time for downclocking, the frame aggregation scheme aims to increase the network throughput and to maintain per-STA temporal fairness. By setting AF in proportion to $R_{\mathrm{tx}}$, each STA occupies a comparable amount of airtime, regardless of its transmission rate, and the network throughput can be increased in multirate WLANs [6].

(2) If the STA does not have a sufficient number of frames to aggregate, that is, $N_{\mathrm{q}}<\left[\alpha\left(R_{\mathrm{tx}} / R_{\min }\right)\right]$, the STA just constructs the aggregated frame with $N_{\mathrm{q}}$ frames without waiting for additional frame arrivals. This approach avoids an unnecessary delay due to frame aggregation.

We need to discuss the effect of $\alpha$ on the performance of the proposed mechanism. As the value of $\alpha$ increases, the downclocking scheme conserves more energy due to IL, and at the same time, the frame aggregation scheme further improves the channel efficiency. However, the longer transmission time due to larger value of $\alpha$ makes the transmission of the aggregated frame liable to be failed due to time-varying channel quality, and it degrades the short-term fairness among STAs. In addition, there exists a limit on the maximum size of aggregated frame as per IEEE 802.11 standard (e.g., $64 \mathrm{~KB}$ ). Taking this trade-off into account, the recommended value of $\alpha$ ranges between one and three.

3.3. Contention Window Control. In addition to the downclocking and frame aggregation schemes, we propose controlling the contention window size of STAs, in order to reduce the ILT unnecessarily spent due to the collision. The ILT in the IEEE 802.11 WLANs is comprised of three parts: (i) time spent in receiving nonintended frame, (ii) time spent in sensing the channel for frame transmission, and (iii) time spent in receiving any collided frame. The first part of ILT can be dealt with the downclocking scheme proposed in Section 3.1, while the second part of ILT can be decreased by the frame aggregation scheme proposed in Section 3.2. If a collision happens between nonintended frames, an overhearing STA cannot switch its state to semisleep because the information required for downclocking cannot be correctly decoded. Otherwise, if an intended frame is involved in the collision, the frame cannot be successfully delivered to the receiving STA and the STA has to sense the channel until the end of collision. Furthermore, the collided frame should be retransmitted, which results in a decrease of throughput and energy efficiency. Therefore, we propose a control scheme of contention window to decrease the ILT due to collision.

The key idea of contention window control is that the collision probability mostly depends on the number of competing STAs and that it can be controlled by the size of the contention window. Note that the IEEE 802.11 standard proposes a minimum value of contention window $\left(\mathrm{CW}_{\min }\right)$ that is not scalable to the number of competing STAs. The IEEE 802.11 standard also proposes the BEB mechanism to compensate for the weakness of static and fixed value of $\mathrm{CW}_{\text {min }}$; with the $\mathrm{BEB}$ mechanism, the value of $\mathrm{CW}_{\min }$ is doubled on detecting the transmission failure so as to mitigate the possible collision in the retransmitted frame. However, the $\mathrm{BEB}$ mechanism is reactive to the transmission failure, so it is not effective to prevent collisions as the number of STAs increases. In addition, it was shown in $[25,26]$ that the optimal value of $\mathrm{CW}_{\min }$ minimizing the collision probability is proportional to the number of STAs. Based on these insights, we propose a proactive approach for controlling the contention window as follows. The AP keeps track of the number of associated STAs, defined as $N_{\text {sta }}$, and advertises this information by periodically broadcasting the beacon frames containing it in the "station count" subfield of "BSS Load" element. After being informed of $N_{\text {sta }}$, each STA sets its contention window, $\mathrm{CW}_{\mathrm{s}}$, as

$$
\mathrm{CW}_{\mathrm{s}}=\beta \mathrm{CW}_{\min } N_{\text {sta }} \text {. }
$$

In (3), $\beta(>0)$ is a scaling factor that can be used to control the trade-off between collision probability and channel access delay. The larger value of $\beta$ leads to the decrease in collision probability at the cost of increase in channel access delay. Note that the STA maintains the BEB mechanism; that is, $\mathrm{CW}_{s}$ is doubled on detecting a transmission failure, which plays as a subsidiary function to deal with collisions.

\section{Analysis Model}

We derive an analysis model to evaluate and compare the performance of the proposed mechanism with those of two conventional approaches, PSM and downclocking. We consider that PSM-enabled STAs (hereafter, the term of "PSM-enabled STA" indicates the STA that implements any type of power saving schemes, for example, IEEE 802.11 PSM, downclocking, or the proposed mechanism) coexist with non-PSM-enabled STAs (background STAs) and that the PSM-enabled STAs receive frames from the AP while background STAs transmit frames to the AP. We consider two performance indices for PSM-enabled STAs, energy per bit and throughput. The former is introduced to measure the energy efficiency and is defined as the amount of energy consumed to successfully retrieve a single bit of data frame, while the latter evaluates the transmission efficiency and is defined as the number of bits successfully delivered during a given time. For the purpose of clarity and tractability, we make the following reasonable assumptions: (i) each STA can sense the transmission of other STAs, such that there is no hidden STA, (ii) the probability of transmission failure resulting from channel error is negligible thanks to an appropriate link adaptation mechanism, (iii) the LI is properly set such that the PSM-enabled STA has data frames to retrieve in the first active BI but goes to sleep during the remaining BIs within the LI, (iv) the background STAs always have data frames to transmit, and (v) the transmission rate of each STA does not change but may be different from that of other STAs. For the tractability of analysis, we consider that the PSMenabled STA always has data frames to retrieve in the active $\mathrm{BIs}$, but no data frame to retrieve in the sleeping BIs. Also, we consider that STAs that have the same transmission rate of $R_{i}$ 
belong to the $i$ th group $(1 \leq i \leq G$, where $G$ is the number of total groups) and that the $i$ th group consists of $n_{i}$ STAs.

In order to derive the analysis model, we employ the twodimensional Markov chain process similarly to the traditional Bianchi's model [25] and its variants [27-29]. Firstly, we calculate the probabilities of channel access, collision, and transmission success. From our previous work in [6], the probability of transmission attempt $\left(P_{\mathrm{tx}, i}\right)$ by an STA in the $i$ th group is given by

$$
\begin{aligned}
P_{\mathrm{tx}, i}= & \frac{1-\left(P_{\mathrm{coll}, i}\right)^{\left(L_{r, i}+1\right)}}{1-P_{\mathrm{coll}, i}} \\
& \times\left[\sum_{k=0}^{L_{r, i}}\left(P_{\mathrm{coll}, i}\right)^{k}\left(1+\frac{W_{k, i}-1}{2\left(1-P_{\mathrm{busy}, i}\right)}\right)\right]^{-1} .
\end{aligned}
$$

Here, $P_{\text {busy }, i}$ and $P_{\text {coll }, i}$ are the probabilities of busy channel and collisions, respectively, $W_{k, i}$ is the contention window size at backoff stage $k$ of group- $i$ STA, and $L_{r, i}$ is the maximum retry limit of group- $i$ STA. Since the group- $i$ STA finds the channel busy if there is at least one ongoing transmission except for itself, $P_{\text {busy }, i}$ can be represented as

$$
P_{\text {busy }, i}=1-\left(1-P_{\mathrm{tx}, i}\right)^{n_{i}-1} \prod_{k=1, k \neq i}^{G}\left(1-P_{\mathrm{tx}, k}\right)^{n_{k}} .
$$

In a similar way, the transmission of the group- $i$ STA collides if more than one STA is transmitting at the same time. Thus, $P_{\text {coll }, i}$ is given as

$$
P_{\mathrm{coll}, i}=1-\left(1-P_{\mathrm{tx}, i}\right)^{n_{i}-1} \prod_{k=1, k \neq i}^{G}\left(1-P_{\mathrm{tx}, k}\right)^{n_{k}} .
$$

It is noteworthy that both $P_{\text {busy, } i}$ and $P_{\text {coll, } i}$ have the same formula, as shown in (5) and (6). However, they occur in different conditions; $P_{\text {busy }, i}$ is the probability under the condition that the group- $i$ STA is not involved in transmission but senses the channel, whereas $P_{\text {coll, } i}$ is the probability under the condition that the group- $i$ STA is transmitting. Let us define $P_{\text {succ, } i}$ as the probability of successful transmission by the group- $i$ STA. If there is exactly one transmission by the group$i$ STA, its transmission is successful, and thus, $P_{\text {succ, } i}$ becomes

$$
P_{\mathrm{succ}, i}=n_{i} P_{\mathrm{tx}, i}\left(1-P_{\mathrm{tx}, i}\right)^{n_{i}-1} \prod_{k=1, k \neq i}^{G}\left(1-P_{\mathrm{tx}, k}\right)^{n_{k}} .
$$

Finally, $P_{\mathrm{tx}, i}, P_{\mathrm{busy}, i}, P_{\mathrm{coll}, i}$, and $P_{\text {succ }, i}$ can be numerically obtained from (4)-(7).

Now, we focus on the performance of power saving mechanisms. A PSM-enabled STA can be in any of the following six states:

(S1) When the channel is idle, all the STAs decrease their backoff counters. This state takes up a small portion of ILT.

(S2) The PSM-enabled STA successfully transmits a PSPoll message and retrieves its data frame from the AP, and then it sends the corresponding ACK frame.
(S3) The PSM-enabled STA overhears nonintended data frames transmitted by background STAs. This state accounts for the major part of ILT.

(S4) There occurs a collision in which the PSM-enabled STA is involved (i.e., the PS-Poll message collides with data frame or other PS-Poll messages).

(S5) There occurs a collision among background STAs. During this state, the PSM-enabled STA unnecessarily consumes its energy in sensing the channel.

(S6) The PSM-enabled STA remains in the sleep state.

By considering these six states, we can obtain the performances of several power saving mechanisms. In the following subsections, we derive the formula of energy per bit and throughput in the cases of the conventional IEEE 802.11 PSM, the downclocking scheme combined with the PSM, and the proposed mechanism.

4.1. Analysis of IEEE 802.11 PSM. Here, we calculate the probability, time, and energy of each state addressed above when the IEEE 802.11 PSM is implemented. Let us denote $N_{\mathrm{ps}}$ and $N_{\mathrm{bg}}$ as the numbers of PSM-enabled STAs and background STAs, respectively, and we denote the total number of STAs as $N_{\text {sta }}=N_{\mathrm{ps}}+N_{\mathrm{bg}}$.

(S1) The probability of backoff state, $P_{\mathrm{bo}, i}$, is given by

$$
P_{\mathrm{bo}, i}=\left(1-P_{\mathrm{tx}, i}\right)^{N_{\mathrm{sta}}} .
$$

We define $T_{\text {slot }}$ as the slot time defined in the IEEE 802.11 standard and $P_{w}^{\mathrm{IL}}$ as the power spent during the ILT. Then, energy consumed during the backoff state, $E_{\mathrm{bo}, i}$, becomes

$$
E_{\mathrm{bo}, i}=P_{\mathrm{bo}, i} P_{w}^{\mathrm{IL}} T_{\text {slot }} \cdot
$$

(S2) The probability of successful data retrieval by the group- $i$ PSM-enabled STA, $P_{s, i}$, is calculated as

$$
P_{s, i}=N_{\mathrm{ps}} P_{\mathrm{tx}, i}\left(1-P_{\mathrm{tx}, i}\right)^{N_{\mathrm{sta}}-1} .
$$

In this state, the PSM-enabled STA transmits a PS-Poll message and ACK frame, as well as receiving data frame from the AP. Thus, the transmission time, $T_{\mathrm{tx}}$, becomes

$$
T_{\mathrm{tx}}=T_{\mathrm{PS}}+T_{\mathrm{ACK}}
$$

Here, $T_{\mathrm{PS}}$ and $T_{\mathrm{ACK}}$ are the time spent in transmitting the PSPoll message and ACK frame, respectively, and they can be represented as

$$
\begin{gathered}
T_{\mathrm{PS}}=T_{\mathrm{PHY}}+\frac{L_{\mathrm{MAC}}+L_{\mathrm{PS}}}{R_{\mathrm{basic}}}, \\
T_{\mathrm{ACK}}=T_{\mathrm{PHY}}+\frac{L_{\mathrm{MAC}}+L_{\mathrm{ACK}}}{R_{\text {basic }}},
\end{gathered}
$$

where $T_{\mathrm{PHY}}$ is the constant time to transmit PHY header, $L_{\mathrm{MAC}}, L_{\mathrm{PS}}$, and $L_{\mathrm{ACK}}$ are the sizes of MAC header, PS-Poll message, and ACK frame in bits, respectively, and $R_{\text {basic }}$ is the transmission rate of ACK frame and PS-Poll message. 
(The transmission rate of ACK frame and PS-Poll message $\left(R_{\text {basic }}\right)$ is usually smaller than the transmission rate of data frame $\left(R_{i}\right)$ for reliable transmission, and it depends on the implementation. In this work, we assume that $R_{\text {basic }}$ is equal to the minimum transmission rate, $R_{\min }$.) In the same way, the time spent in receiving the data frame from the AP, $T_{\mathrm{rx}, i}$, is represented as

$$
T_{\mathrm{rx}, i}=T_{\mathrm{DIFS}}+2 T_{\mathrm{SIFS}}+T_{\mathrm{PHY}}+\frac{L_{\mathrm{MAC}}+L_{\mathrm{frm}}}{R_{i}},
$$

where $L_{\text {frm }}$ is the size of frame transmitted by the AP. Defining $P_{w}^{\mathrm{RX}}$ and $P_{w}^{\mathrm{TX}}$ as the receiving and transmitting power, respectively, the total energy consumed in this state, $E_{s, i}$, becomes

$$
E_{s, i}=P_{s, i}\left(P_{w}^{\mathrm{TX}} T_{\mathrm{tx}}+P_{w}^{\mathrm{RX}} T_{\mathrm{rx}, i}\right) .
$$

(S3) This state is identical to the case where a background STA successfully transmits its frame without any collision. The probability of this state, $P_{s, i}^{\mathrm{bg}}$, can be represented as

$$
P_{s, i}^{\mathrm{bg}}=N_{\mathrm{bg}} P_{\mathrm{tx}, i}\left(1-P_{\mathrm{tx}, i}\right)^{N_{\mathrm{sta}}-1} .
$$

The duration of this state, denoted as $T_{\mathrm{bg}}$, is

$$
T_{\mathrm{bg}}=T_{\mathrm{DIFS}}+T_{\mathrm{PHY}}+\frac{L_{\mathrm{MAC}}+L_{\mathrm{frm}}}{R_{\mathrm{bg}}}+T_{\mathrm{SIFS}}+T_{\mathrm{ACK}} \text {, }
$$

where $R_{\mathrm{bg}}$ is the transmission rate of the background STA. The energy consumption of the PSM-enabled STA in overhearing the nonintended frame, $E_{s, i}^{\mathrm{bg}}$, is

$$
E_{s, i}^{\mathrm{bg}}=P_{s, i}^{\mathrm{bg}} P_{w}^{\mathrm{RX}} T_{\mathrm{bg}}
$$

(S4) There are two substates of collision where the PSMenabled STA is involved. The first one is the collision between two or more PS-Poll messages; the second one is the collision between PS-Poll message and data frame. The corresponding probabilities of these two substates, denoted as $P_{c, i}^{1}$ and $P_{c, i}^{2}$, respectively, can be obtained as

$$
\begin{aligned}
P_{c, i}^{1}= & \left(1-\left(1-P_{\mathrm{tx}, i}\right)^{N_{\mathrm{ps}}}-N_{\mathrm{ps}} P_{\mathrm{tx}, i}\left(1-P_{\mathrm{tx}, i}\right)^{N_{\mathrm{ps}}-1}\right) \\
& \times\left(1-P_{\mathrm{tx}, i}\right)^{N_{\mathrm{bg}}} \\
P_{c, i}^{2}= & \left(1-\left(1-P_{\mathrm{tx}, i}\right)^{N_{\mathrm{ps}}}\right)\left(1-\left(1-P_{\mathrm{tx}, i}\right)^{N_{\mathrm{bg}}}\right) .
\end{aligned}
$$

In the first case, the PSM-enabled STA consumes energy in transmitting the PS-Poll message; in the second case, it consumes additional energy in overhearing the collided data frame. Therefore, the effective energy consumed by the PSM-enabled STA during this state can be represented as

$$
\begin{aligned}
E_{c, i}= & P_{c, i}^{1} P_{w}^{\mathrm{TX}} T_{\mathrm{PS}} \\
& +P_{c, i}^{2}\left(P_{w}^{\mathrm{TX}} T_{\mathrm{PS}}+P_{w}^{\mathrm{RX}}\left(T_{\mathrm{bg}}-T_{\mathrm{PS}}-T_{\mathrm{ACK}}\right)\right) .
\end{aligned}
$$

(S5) The probability of collision between background STAs, $P_{c, i}^{\mathrm{bg}}$, is

$$
\begin{aligned}
P_{c, i}^{\mathrm{bg}}= & \left(1-\left(1-P_{\mathrm{tx}, i}\right)^{N_{\mathrm{bg}}}-N_{\mathrm{bg}} P_{\mathrm{tx}, i}\left(1-P_{\mathrm{tx}, i}\right)^{N_{\mathrm{bg}}-1}\right) \\
& \times\left(1-P_{\mathrm{tx}, i}\right)^{N_{\mathrm{ps}}},
\end{aligned}
$$

and the energy consumption of PSM-enabled STA in overhearing the collided data frame, $E_{c, i}^{\mathrm{bg}}$, becomes

$$
E_{c, i}^{\mathrm{bg}}=P_{c, i}^{\mathrm{bg}} P_{w}^{\mathrm{RX}}\left(T_{\mathrm{bg}}-T_{\mathrm{ACK}}\right) .
$$

It is important to note that all the probabilities of active states in (8), (10), (15), (18), and (20) are calculated for those BIs where all the PSM-enabled STAs are active and that these probabilities of group- $i$ STAs are characterized by $P_{\mathrm{tx}, i}$, which can be obtained numerically from (4) to (7) by considering the probabilities of all the other groups.

(S6) We define $P_{w}^{\mathrm{SL}}$ as the amount of power consumed in the sleep state and $L$ as a positive integer value such that $L I=$ $L \times \mathrm{BI}$. We define a virtual cycle as the time interval between the time at which a certain PSM-enabled STA starts a backoff procedure to retrieve a frame from the AP and the time at which it starts the next backoff procedure within an active BI. Thus, the virtual cycle consists of several times spent in each active state (S1)-(S5) except for the sleep state (S6), and its average value for the group- $i \mathrm{STA}, T_{\mathrm{vc}, i}$, can be represented as

$$
\begin{aligned}
T_{\mathrm{vc}, i}= & P_{\mathrm{bo}, i} T_{\mathrm{slot}}+P_{s, i}\left(T_{\mathrm{tx}}+T_{\mathrm{rx}, i}\right)+P_{s, i}^{\mathrm{bg}} T_{\mathrm{bg}}+P_{c, i}^{1} T_{\mathrm{PS}} \\
& +\left(P_{c, i}^{2}+P_{c, i}^{\mathrm{bg}}\right)\left(T_{\mathrm{bg}}-T_{\mathrm{ACK}}\right) .
\end{aligned}
$$

Note that the PSM-enabled STA can retrieve frames from the AP several times within one active $\mathrm{BI}$ and the ratio of time required for retrieving one frame to the whole active BI is $T_{\mathrm{vc}, i} / \mathrm{BI}$. Then, the effective sleep time spent in retrieving one frame by the group- $i$ PSM-enabled STA, $T_{\text {slp }, i}$, and the corresponding energy consumption in the sleep state, $E_{\text {slp }, i}$, become

$$
\begin{aligned}
& T_{\mathrm{slp}, i}=\frac{T_{\mathrm{vc}, i}}{\mathrm{BI}}(L-1) \mathrm{BI}=(L-1) T_{\mathrm{vc}, i}, \\
& E_{\mathrm{slp}, i}=T_{\mathrm{slp}, i} P_{w}^{\mathrm{SL}} .
\end{aligned}
$$

Now, we define the energy per bit $\left(E_{\mathrm{b}, i}\right)$ as the effective amount of energy consumed to successfully retrieve a single bit of data frame; that is,

$$
E_{\mathrm{b}, i}=\frac{E_{\mathrm{total}, i}}{P_{s, i} L_{\mathrm{frm}}}
$$

where $E_{\text {total, } i}$ is the total energy consumed for the PSMenabled STA to retrieve one frame from the AP; that is,

$$
E_{\mathrm{total}, i}=E_{\mathrm{bo}, i}+E_{s, i}+E_{s, i}^{\mathrm{bg}}+E_{c, i}+E_{c, i}^{\mathrm{bg}}+E_{\mathrm{slp}, i} .
$$

We denote $T_{\mathrm{th}, i}$ as the throughput achieved by the group- $i$ PSM-enabled STA, which is the number of bits successfully transmitted during a virtual cycle including sleep state, that is, $L \cdot T_{\mathrm{vc}, i}$. Then, $T_{\mathrm{th}, i}$ can be represented as

$$
T_{\mathrm{th}, i}=\frac{1}{n_{i}}\left(\frac{P_{s, i} L_{\mathrm{frm}}}{L \cdot T_{\mathrm{vc}, i}}\right) .
$$


4.2. Analysis of Downclocking Scheme. From the view point of energy efficiency, the downclocking scheme is identical to the IEEE 802.11 PSM, except for the reduced power in overhearing nonintended frames destined to the background STAs. The probabilities of each of the six states do not change from those of IEEE 802.11 PSM; the only difference happens in the energy consumption at the state (S3). Similar to (1), the semisleep time at the state (S3) is given as $T_{\mathrm{ss}}=T_{\mathrm{bg}}-2 T_{\mathrm{tran}}-$ $T_{\mathrm{h}}$. Here, we assume that $T_{\mathrm{bg}}$ is large enough such that $T_{\mathrm{ss}}$ has a positive value. Let us denote $P_{w}^{\mathrm{SS}}$ as the power during the semisleep time, which is less than $P_{w}^{\mathrm{RX}}$ due to downclocking. Then, the energy consumption at state (S3), $E_{s, i}^{\mathrm{bg}}$ in (17), is changed as

$$
E_{s, i}^{\mathrm{bg}}=P_{s, i}^{\mathrm{bg}}\left(P_{w}^{\mathrm{RX}}\left(T_{\mathrm{bg}}-T_{\mathrm{ss}}\right)+P_{w}^{\mathrm{SS}} T_{\mathrm{ss}}\right)
$$

It is important to note that the throughput of downclocking scheme does not change from that of IEEE 802.11 PSM.

4.3. Analysis of the Proposed Mechanism. Compared to the downclocking scheme, the proposed mechanism maintains two additional features of frame aggregation and contention window control. Note that the frame aggregation and contention window control are applied to all the STAs, regardless of the capability of PSM. In order to get the analysis results for the proposed mechanism, several probabilities represented in (4)-(7) need to be obtained numerically by considering the change in the minimum contention value $\left(W_{k, i}\right)$ as given in (3). This leads to the different probabilities of all the six states from those of IEEE 802.11 PSM and downclocking. Moreover, we should consider the frame aggregation as given in (2) when calculating the transmission time of aggregated frame. Let us define $\mathrm{AF}_{i}$ as the number of aggregated frames that are delivered to a group- $i$ PSM-enabled STA after it successfully transmits a PS-Poll message to the AP. With the traffic model considered in this analysis model, $\mathrm{AF}_{i}$ at state (S2) becomes $\mathrm{AF}_{i}=\left[\alpha\left(R_{i} / R_{\mathrm{min}}\right)\right]$ and $T_{\mathrm{rx}, i}$ needs to be changed from (13) to

$$
\begin{aligned}
T_{\mathrm{rx}, i}= & T_{\mathrm{DIFS}}+2 T_{\mathrm{SIFS}}+T_{\mathrm{PHY}}+\frac{\mathrm{AF}_{i}\left(L_{\mathrm{MAC}}+\mathrm{L}_{\mathrm{frm}}\right)}{R_{i}} \\
= & T_{\mathrm{DIFS}}+2 T_{\mathrm{SIFS}}+T_{\mathrm{PHY}} \\
& +\left[\alpha \frac{R_{i}}{R_{\mathrm{min}}}\right] \frac{L_{\mathrm{MAC}}+L_{\mathrm{frm}}}{R_{i}} .
\end{aligned}
$$

In the same way, the transmission time of background STAs at states (S3), (S4), and (S5) is also changed due to frame aggregation as

$$
\begin{aligned}
T_{\mathrm{bg}}= & T_{\mathrm{DIFS}}+T_{\mathrm{PHY}}+\frac{\mathrm{AF}_{\mathrm{bg}}\left(L_{\mathrm{MAC}}+L_{\mathrm{frm}}\right)}{R_{\mathrm{bg}}}+T_{\mathrm{SIFS}} \\
& +T_{\mathrm{BACK}} \cdot
\end{aligned}
$$

Here, $\mathrm{AF}_{\mathrm{bg}}$ is the number of aggregated frames for a background STA to transmit; that is, $\mathrm{AF}_{\mathrm{bg}}=\left[\alpha\left(R_{\mathrm{bg}} / R_{\min }\right)\right]$;
TABLE 1: IEEE 802.11 PHY/MAC parameters used in simulations.

\begin{tabular}{lc}
\hline Parameters & Value \\
\hline Idle listening power $\left(P_{w}^{\mathrm{IL}}\right)$ & $0.9 \mathrm{~W}$ \\
\hline Receiving power $\left(P_{w}^{\mathrm{RX}}\right)$ & $0.9 \mathrm{~W}$ \\
\hline Transmission power $\left(P_{w}^{\mathrm{TX}}\right)$ & $1.4 \mathrm{~W}$ \\
\hline Semisleep power $\left(P_{w}^{\mathrm{SS}}\right)$ & $0.45 \mathrm{~W}$ \\
\hline Sleep power $\left(P_{w}^{\mathrm{SL}}\right)$ & $0.06 \mathrm{~W}$ \\
\hline frame size $\left(L_{\text {frm }}\right)$ & 1500 bytes \\
\hline $\begin{array}{l}\text { Size of MAC header with MPDU } \\
\text { subframe header }\left(L_{\mathrm{MAC}}\right)\end{array}$ & 28 bytes \\
\hline Size of block ACK frame $\left(L_{\mathrm{BACK}}\right)$ & 30 bytes \\
\hline Size of ACK frame $\left(L_{\mathrm{ACK}}\right)$ & 14 bytes \\
\hline Minimum transmission rate $\left(R_{\min }\right)$ & $6 \mathrm{Mb} / \mathrm{s}$ \\
\hline $\begin{array}{l}\text { Transmission time of PHY preamble and } \\
\text { header }\left(T_{\mathrm{PHY}}\right)\end{array}$ & $20 \mu \mathrm{s}$ \\
\hline$T_{\mathrm{DIFS}}, T_{\mathrm{SIFS}}$ & $28,10 \mu \mathrm{s}$ \\
\hline Slot time $\left(T_{\text {slot }}\right)$ & $9 \mu \mathrm{s}$ \\
\hline $\begin{array}{l}\text { Minimum } / \text { maximum size of contention } \\
\text { window }(\mathrm{CW} \\
\text { min }\end{array} / \mathrm{CW}$ max
\end{tabular}

$T_{\mathrm{BACK}}$ is the time required for transmitting the block ACK frame; that is,

$$
T_{\mathrm{BACK}}=T_{\mathrm{PHY}}+\frac{L_{\mathrm{MAC}}+L_{\mathrm{BACK}}}{R_{\text {basic }}},
$$

where $L_{\mathrm{BACK}}$ is the size of block ACK frame. In addition to $T_{\mathrm{rx}, i}$ and $T_{\mathrm{bg}}, T_{\mathrm{ACK}}$ in (11), (19), (21), and (22) needs to be replaced with $T_{\mathrm{BACK}}$. Also, $L_{\text {frm }}$ in (24) and (26) is replaced with $\mathrm{AF}_{i} \cdot L_{\text {frm }}$ to account for the frame aggregation. The effect of downclocking can also be considered from (27) with the extended semisleep time, $T_{\mathrm{ss}}$, due to the change of $T_{\mathrm{bg}}$ in (29). The remaining equations can be calculated straightforwardly, as in Section 4.1.

\section{Simulation Results and Discussion}

5.1. Simulation Configuration. In this section, we validate the analysis model and compare the performance of the proposed mechanism with those of the conventional power saving mechanisms. We developed our simulator using MATLAB, which implements the IEEE 802.11g MAC/PHY layers. Table 1 lists the parameters and their values used in the simulations. The values of power consumption in Table 1 were set based on the real measurements of the WLAN interface card [30] (we performed the simulations with a different set of power consumption parameters given in a recent paper [31] and found that the relative performance is hardly affected by these parameters). In the proposed mechanism, we set two scaling factors, $\alpha$ and $\beta$, as 1 . In order to focus on the efficiency of power saving mechanisms, we consider only downlink traffic for the PSM-enabled STAs and uplink traffic for the background STAs. If we consider uplink traffic in the PSMenabled STAs, as well as downlink traffic, we expect that the energy efficiency will decrease with respect to the load of uplink traffic; however, the relative performance among 


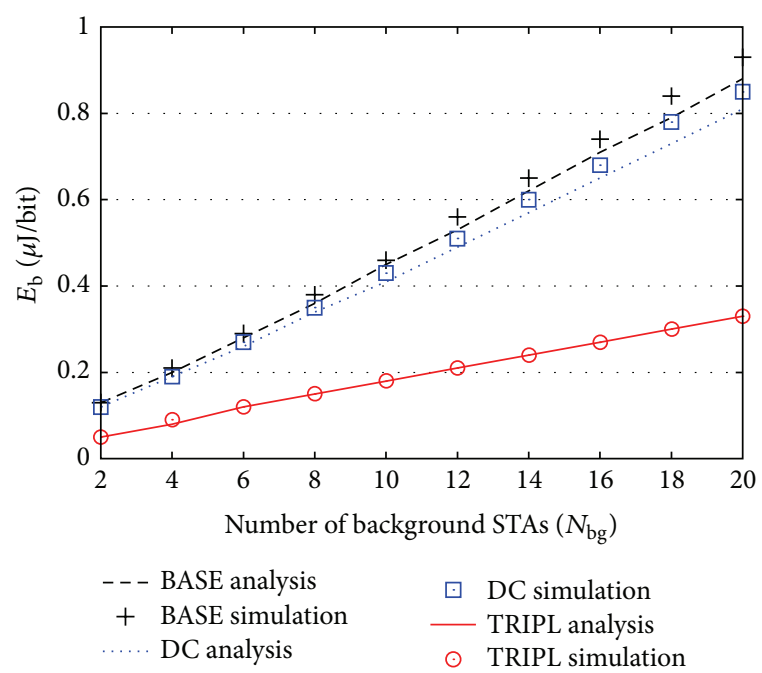

(a) Energy per bit $\left(E_{\mathrm{b}}\right)$

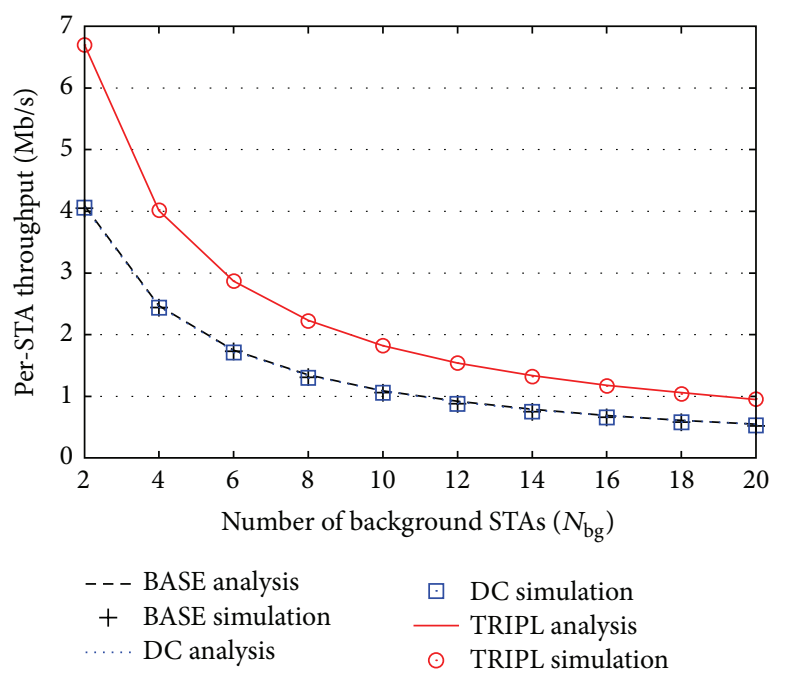

(b) Per-STA throughput of PSM-enabled STAs $\left(\mathrm{TH}_{\mathrm{STA}}\right)$

FIgURE 3: Comparison of analysis and simulation results for BASE, DC, and TRIPL (Scenario 1).

all the comparative mechanisms will be mostly maintained because the PSM-enabled STAs work in the same way to transmit the uplink traffic, regardless of power saving schemes. Also, the effect of uplink traffic in the PSM-enabled STAs can be indirectly inferred from the effect of increasing in the number of background STAs. We set the beacon interval to $100 \mathrm{~ms}$, which is the default value recommended by the IEEE 802.11 standard, and set the simulation time to 1 million time slots. We consider the following four scenarios in terms of the number of STAs, transmission rate, and traffic load, to evaluate the performance of the proposed mechanism under diverse configurations.

Scenario 1. We fix $N_{\mathrm{ps}}$ as 1 while varying $N_{\mathrm{bg}}$ from 2 to 20. All the STAs have the same transmission rate of $48 \mathrm{Mb} / \mathrm{s}$. In addition, we set LI of PSM-enabled STA to two beacon intervals and consider that the PSM-enabled STA attempts to retrieve as many frames as possible during the first beacon interval within the LI and goes to sleep during the next beacon interval, while background STAs always have data frame to transmit.

Scenario 2. We set both $N_{\mathrm{ps}}$ and $N_{\mathrm{bg}}$ to 4 and set the transmission rate of each STA differently, to $6,12,24$, and $48 \mathrm{Mb} / \mathrm{s}$. We consider the same traffic model as Scenario 1. The LI is set to two beacon intervals for all four PSM-enabled STAs, but the active beacon intervals for frame retrieval are set differently among them.

Scenario 3. We set the interarrival time of frames for all the STAs randomly according to an exponential distribution with the mean value of $50 \mathrm{~ms}$. Here, we set LI as three beacon intervals and set $N_{\mathrm{ps}}$ as 1 while increasing $N_{\mathrm{bg}}$ from 2 to 20 .

Scenario 4. We consider a configuration of light traffic load where the frame arrivals per beacon interval follow a Poisson distribution with the mean value ranging from 1 to 10 . We fix $N_{\mathrm{ps}}$ as 1 and consider several values of $N_{\mathrm{bg}}$, such as 4,6 , and 10 .

As the performance indices, we consider energy per bit and throughput, which represent the energy efficiency and transmission efficiency, respectively. All the values of performance indices given at tables and figures in this section represent the averaged value with 30 instances of simulations.

5.2. Validation of Analysis Model. In the first simulation, we validate the analysis model derived in Section 4, by comparing the analysis results with simulation results. We consider three power saving mechanisms in Section 4; hereafter, we refer to them as BASE (IEEE 802.11 PSM), DC (downclocking), and TRIPL (proposed mechanism). The simulations were conducted for Scenario 1. Figure 3(a) confirms the accuracy of analysis models; that is, the energy per bit $\left(E_{\mathrm{b}}\right)$ attained from simulations closely coincides with that obtained from analysis. As shown in Figure 3(a), $E_{\mathrm{b}}$ increases with respect to the increase of $N_{\mathrm{bg}}$ due to the increase of ILT and collision. As $N_{\text {bg }}$ increases from 2 to $20, E_{\mathrm{b}}$ values of BASE, DC, and TRIPL, increases from $0.13,0.12$, and 0.05 , to $0.93,0.85$, and 0.33 in $\mu \mathrm{J} / \mathrm{bit}$, respectively. It is worthwhile to note that the effect of downclocking on energy efficiency is not drastic. Compared to BASE, DC lowers $E_{\mathrm{b}}$ by about $8 \%$. However, TRIPL significantly decreases $E_{\mathrm{b}} 2.6$ - and 2.4-fold on average, compared to BASE and DC, respectively.

Moreover, we validate the analysis results in terms of perSTA throughput of PSM-enabled STA $\left(\mathrm{TH}_{\mathrm{STA}}\right)$ in Figure 3(b). We observe from Figure 3(b) that there is no significant deviation between simulation results and analysis results. Recall that the analysis model gives absolutely no difference in $\mathrm{TH}_{\mathrm{STA}}$ of BASE and DC. Figure 3(b) shows that $\mathrm{TH}_{\mathrm{STA}}$ decreases as $N_{b g}$ increases for all the mechanisms. This is because all the STAs share the channel, and the probability of collision increases as $N_{\text {bg }}$ increases. TRIPL maintains a higher 
TABLE 2: Simulation results in terms of energy per bit $\left(E_{\mathrm{b}}\right)$ and the fraction of energy consumption due to idle listening $\left(\gamma_{\mathrm{IL}}\right)$ under multirate environment (Scenario 2).

\begin{tabular}{lccccc}
\hline \multirow{2}{*}{ STA ID } & $R_{\mathrm{tx}}(\mathrm{Mb} / \mathrm{s})$ & \multicolumn{2}{c}{$\mathrm{BASE}$} & \multicolumn{2}{c}{ TRIPL } \\
& & $E_{\mathrm{b}}(\mu \mathrm{J} / \mathrm{bit})$ & $\gamma_{\mathrm{IL}}(\%)$ & $E_{\mathrm{b}}(\mu \mathrm{J} / \mathrm{bit})$ & $\gamma_{\mathrm{IL}}(\%)$ \\
\hline $\mathrm{STA}_{1}$ & 6 & 0.82 & 63.35 & 0.80 & 64.69 \\
$\mathrm{STA}_{2}$ & 12 & 0.83 & 75.19 & 0.40 & 64.77 \\
$\mathrm{STA}_{3}$ & 24 & 0.59 & 77.03 & 0.20 & 64.66 \\
$\mathrm{STA}_{4}$ & 48 & 0.58 & 80.56 & 0.10 & 64.59 \\
\hline
\end{tabular}

value of $\mathrm{TH}_{\mathrm{STA}}$ than BASE and DC by about $65-85 \%$, which results from the decrease of collision and channel access overhead.

5.3. Performance Validation of the Proposed Mechanism. The goal of this simulation is to compare the performance of TRIPL with BASE when several PSM-enabled STAs coexist with background STAs and STAs have different transmission rates. Table 2 lists energy per bit $\left(E_{\mathrm{b}}\right)$ and the fraction of energy consumption due to IL $\left(\gamma_{\mathrm{IL}}\right)$ for four PSM-enabled STAs $\left(\mathrm{STA}_{1}-\mathrm{STA}_{4}\right)$ under Scenario 2. The transmission rate given in Table 2 indicates the transmission rate of data frame from the AP to each PSM-enabled STA. Note that STA 1 and $\mathrm{STA}_{2}$ retrieve data frames at the odd beacon intervals and sleep at the even beacon intervals, while $\mathrm{STA}_{3}$ and $\mathrm{STA}_{4}$ alternate data retrieval and sleep in different beacon intervals as compared to $\mathrm{STA}_{1}$ and $\mathrm{STA}_{2}$.

First, we investigate the performance of BASE. In terms of $E_{\mathrm{b}}$, all four STAs have similar performance in spite of the difference in the transmission rate as long as they share the same beacon intervals for the active state: $E_{\mathrm{b}}$ of $\mathrm{STA}_{1}$ and $\mathrm{STA}_{2}$ is about $0.82 \mu \mathrm{J} /$ bit while $E_{\mathrm{b}}$ of $\mathrm{STA}_{3}$ and $\mathrm{STA}_{4}$ is about $0.59 \mu \mathrm{J} / \mathrm{bit}$. These results can be explained as follows. According to the operation of CSMA in multirate WLANs, the PSM-enabled STAs with the same active beacon interval receive a comparable amount of data frames, regardless of the transmission rates. Meanwhile, they have the same power in receiving intended frames or in overhearing nonintended frames. On the other hand, $E_{\mathrm{b}}$ of $\mathrm{STA}_{3}$ and $\mathrm{STA}_{4}$ is decreased compared to that of $\mathrm{STA}_{1}$ and $\mathrm{STA}_{2}$; that is, the energy efficiency is improved in high-rate STAs because they can retrieve more data in a given time. However, it is important to note in Table 2 that even though $E_{\mathrm{b}}$ of BASE decreases in high-rate STAs, $\gamma_{\mathrm{IL}}$ rather increases with respect to the increase of transmission rate. This means that high-rate STAs dissipate more energy due to the ILT. When high-rate STAs coexist with low-rate STAs, high-rate STAs spend more ILT in overhearing nonintended frames destined to low-rate STAs that occupy more airtime to receive a data frame than those destined to high-rate STAs.

Next, we focus on the performance of TRIPL. The results of TRIPL are quite different from those of BASE and are summarized as follows:

(1) While $E_{\mathrm{b}}$ of BASE is independent of transmission rate for STAs sharing the same active beacon intervals, $E_{\mathrm{b}}$ of TRIPL is inversely proportional to the transmission rate, regardless of STA's active beacon interval.

(2) The increase of transmission rate increases $\gamma_{\mathrm{IL}}$ of BASE, but it hardly affects $\gamma_{\mathrm{IL}}$ of TRIPL.

(3) Compared to BASE, TRIPL decreases $E_{\mathrm{b}}$ by up to almost 6 times and decreases $\gamma_{\mathrm{IL}}$ by up to $16 \%$.

These results mainly stem from the frame aggregation implemented in TRIPL, which makes all the STAs occupy the comparable amount of airtime, regardless of their transmission rates. Each STA consumes a comparable amount of energy in overhearing nonintended frames, regardless of transmission rate, but the amount of bits delivered per channel access is proportional to the transmission rate. Therefore, $E_{\mathrm{b}}$ becomes inversely proportional to transmission rate but $\gamma_{\mathrm{IL}}$ becomes immune to transmission rate. As well as the frame aggregation, the downclocking and contention window control in TRIPL contribute to decreasing $E_{\mathrm{b}}$ and $\gamma_{\mathrm{IL}}$.

5.4. Performance Comparison. In this section, we compare the performance of TRIPL with several mechanisms via simulations. As well as BASE and DC, we consider another two mechanisms, FA and DUAL. The former implements only the frame aggregation scheme as described in Section 3.2, whereas the latter integrates two schemes of downclocking and frame aggregation as proposed in Sections 3.1 and 3.2, respectively. By comparing the performances of DC, FA, and DUAL, we can quantitatively evaluate how large a contribution the downclocking or frame aggregation makes to improving energy/transmission efficiency. In the same way, we can observe the effect of contention window control by comparing the performances of DUAL and TRIPL. In this section, we consider two types of traffic models. With the first traffic model, all the PSM-enabled STAs always have data frames to retrieve in the active beacon intervals and all the background STAs always have data frames to transmit. Meanwhile, with the second traffic model, the PSM-enabled STAs sometimes do not have data frames to retrieve, even in the active beacon intervals, and switch to the sleep state, and the background STAs do not always have data frames to transmit. We refer to these two types of traffic as heavy traffic and light traffic and consider them in the subsequent Sections 5.4.1 and 5.4.2, respectively.

5.4.1. Performance Comparison under Heavy Traffic. Figure 4(a) compares energy per bit $\left(E_{\mathrm{b}}\right)$ of several mechanisms under Scenario 1. As shown in Figure $4(\mathrm{a}), E_{\mathrm{b}}$ of all the mechanisms linearly increases as $N_{\mathrm{bg}}$ increases. The increase of $E_{\mathrm{b}}$ is primarily due to the aggravation of contention, collision, and IL with respect to the increase of $N_{\mathrm{bg}}$. We observe the following results from Figure 4(a):

(1) When $N_{\mathrm{bg}}$ is small, $E_{\mathrm{b}}$ of DC is not much decreased from that of BASE; that is, the downclocking cannot effectively improve energy efficiency. This is because the gain of downclocking is realized when the PSMenabled STA overhears nonintended frames.

(2) For the entire range of $N_{b g}, E_{b}$ of FA is lower than that of BASE and DC by about $33 \%$ and $28 \%$, respectively. 


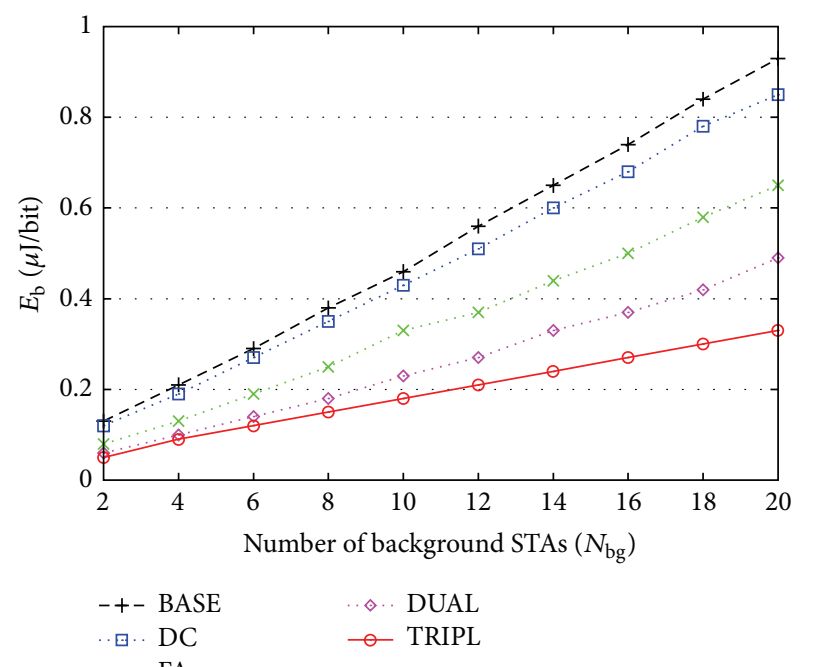

(a) Energy per bit $\left(E_{\mathrm{b}}\right)$

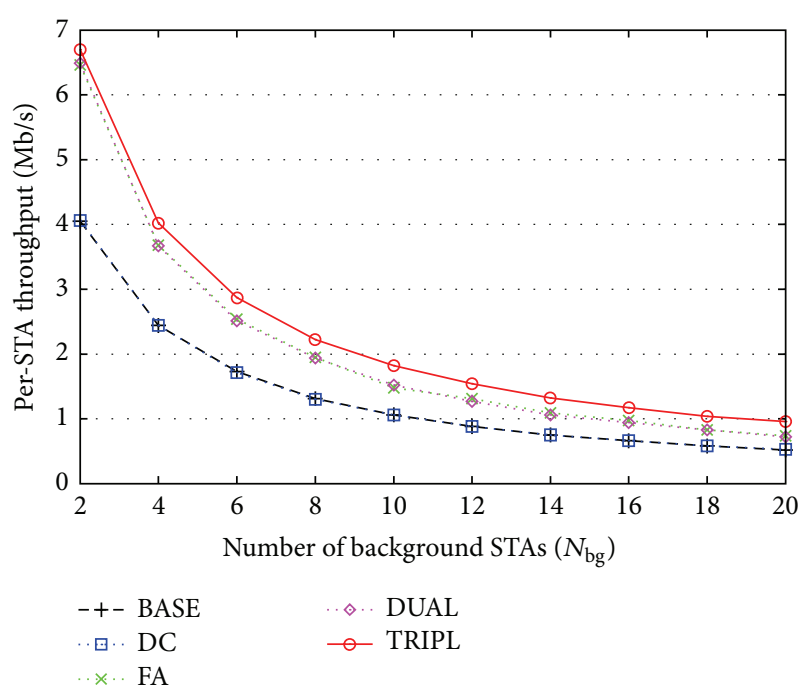

(b) Per-STA throughput of PSM-enabled STAs $\left(\mathrm{TH}_{\mathrm{STA}}\right)$

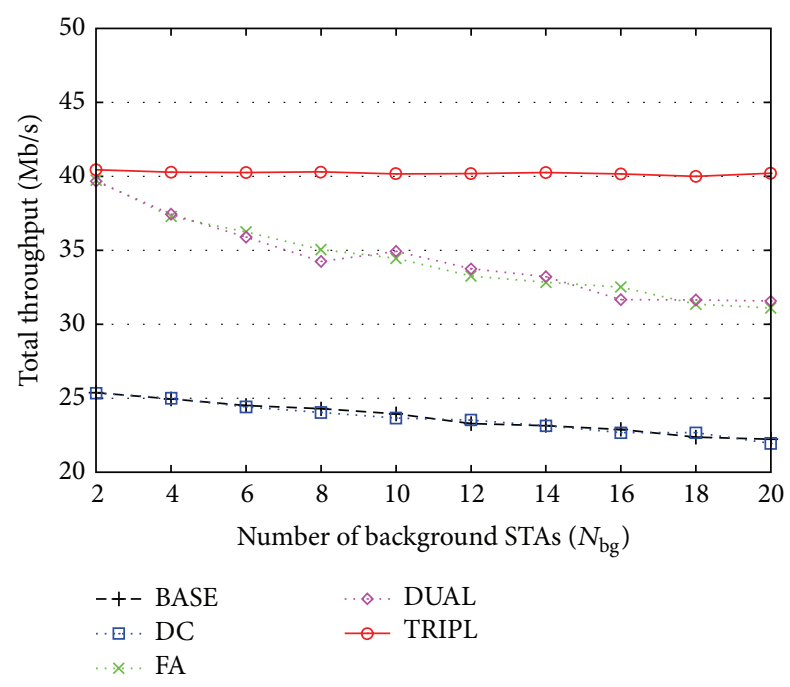

(c) Total throughput $\left(\mathrm{TH}_{\mathrm{TOTAL}}\right)$

FIGURE 4: Performance comparison of TRIPL with different mechanisms (Scenario 1).
This means that the frame aggregation without downclocking saves more energy than the downclocking without frame aggregation, reconfirming the limited gain of the downclocking.

(3) The combination of downclocking and frame aggregation amplifies the synergy in improving energy efficiency; on average, $E_{\mathrm{b}}$ of DC and FA is lower than that of BASE by about $8 \%$ and $33 \%$, respectively, but $E_{\mathrm{b}}$ of DUAL is lower than that of BASE by at least $61 \%$.

(4) As $N_{b g}$ increases, the gain of contention window control increases. As long as $N_{\mathrm{bg}}<10, E_{\mathrm{b}}$ of TRIPL is smaller than that of DUAL by about $16 \%$; however, it becomes smaller by up to $33 \%$ when $N_{\text {bg }}=20$. This confirms that the contention window control is effective in mitigating collision and avoiding unnecessary retransmission, which become severe when $N_{\text {sta }}$ is large.

(5) TRIPL significantly outperforms the other mechanisms; compared to BASE, DC, and FA, it reduces $E_{\mathrm{b}}$ by up to $2.9,2.6$, and 2.0 times, respectively. TRIPL systematically combines three individual mechanisms of downclocking, frame aggregation, and contention window control, each of which effectively handles energy wastage due to nonintended frame, channel access, and collision, respectively.

Next, we observe and compare per-STA throughput of PSM-enabled STA $\left(\mathrm{TH}_{\mathrm{STA}}\right)$ of several mechanisms in Figure 4(b). As expected, DC achieves almost the same throughput as BASE because the downclocking hardly affects throughput. For the same reason, there is little difference between $\mathrm{TH}_{\mathrm{STA}}$ of FA and $\mathrm{TH}_{\mathrm{STA}}$ of DUAL. Figure 4(b) shows that $\mathrm{TH}_{\mathrm{STA}}$ of FA (or DUAL) is higher than that of BASE (or DC) by about $31 \%$; this increase results from the decrease of channel access overhead (i.e., backoff, header overhead, and ACK transmission) due to frame aggregation. In Figure 4(b), it is interesting to observe that (i) when $N_{\text {bg }}$ is small, $\mathrm{TH}_{\text {STA }}$ of DUAL is quite larger than that of BASE but slightly smaller than that of TRIPL; (ii) as $N_{\mathrm{bg}}$ increases, TRIPL further outperforms DUAL in terms of $\mathrm{TH}_{\mathrm{STA}}$; that is, the ratio of $\mathrm{TH}_{\mathrm{STA}}$ of TRIPL to that of DUAL increases from 1.03 to 1.33 when $N_{\text {bg }}$ increases from 2 to 20 . These results imply that the frame aggregation plays an important role in increasing throughput when there are few STAs, whereas the contention window control does so when there are many STAs. Since TRIPL adopts both schemes, it maintains the outstanding performance of $\mathrm{TH}_{\mathrm{STA}}$, regardless of $\mathrm{N}_{\mathrm{bg}}$; TRIPL attains $\mathrm{TH}_{\mathrm{STA}}$ higher than BASE (DC) and FA (DUAL) by up to $85 \%(81 \%)$ and $30 \%$ (33\%), respectively.

We can observe an interesting result in Figure 4(c) that the total throughput $\left(\mathrm{TH}_{\text {TOTAL }}\right)$ of TRIPL is almost immune to the increase of $N_{b g}$, but other mechanisms suffer from the decrease of total throughput with respect to the increase of $N_{\text {bg }}$. The robust performance of TRIPL in response to the change of $N_{\text {bg }}$ stems from the fact that TRIPL effectively mitigates collision by adjusting the minimum contention window with respect to the number of STAs. 


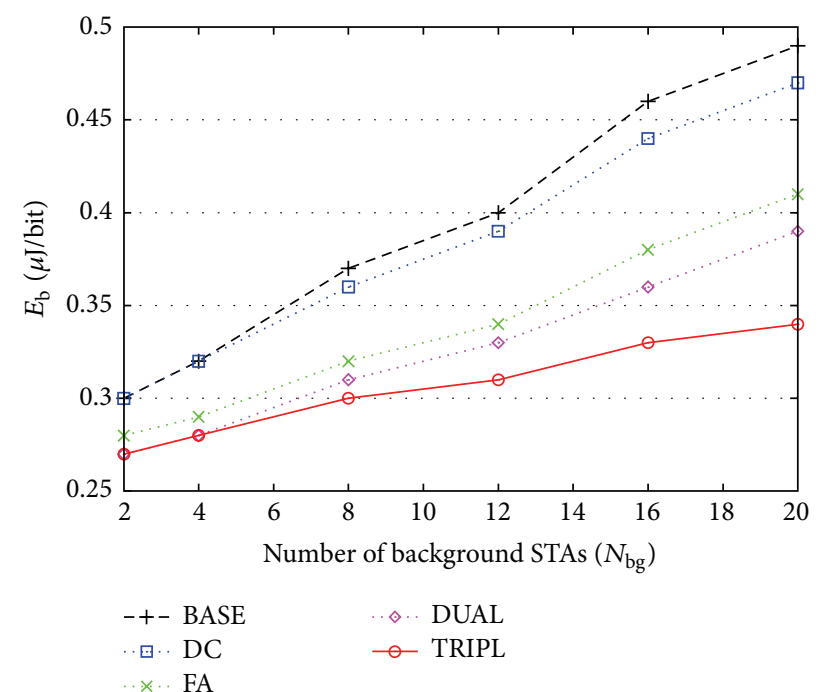

FIGURE 5: Energy efficiency of several mechanisms with light traffic load (Scenario 3).

5.4.2. Performance Comparison under Light Traffic. Up to now, we have investigated the performance of several mechanisms with heavy traffic under Scenarios 1 and 2. Now, we will consider light traffic under Scenarios 3 and 4.

Figure 5 shows the results of $E_{\mathrm{b}}$ for several mechanisms under Scenario 3. Here, the average interarrival time of frames is set to $50 \mathrm{~ms}$ according to an exponential distribution; that is, two frames arrive during a beacon interval on average. As shown in Figure 5, the increase of $N_{\mathrm{bg}}$ mostly increases $E_{\mathrm{b}}$ for all the mechanisms. Compared to the values of $E_{\mathrm{b}}$ in Figure 4(a), the values of $E_{\mathrm{b}}$ in Figure 5 are reduced as a whole, because the PSM-enabled STA can switch to the sleep state when there is no frame to retrieve from the AP and the energy consumption due to IL is decreased. Among all the mechanisms, TRIPL maintains the lowest value of $E_{\mathrm{b}}$, less than that of BASE, DC, FA, and DUAL by up to $31 \%, 28 \%$, $17 \%$, and $13 \%$, respectively. Although TRIPL still outperforms the other mechanisms notably, the relative gain of TRIPL over other mechanisms is slightly reduced, compared to the case of heavy traffic (see Figure 4(a)). This is because the advantage of frame aggregation cannot be fully obtained when the traffic load is light; that is, the semisleep time decreases but the ILT due to channel access increases accordingly.

We further investigate the effect of traffic load on the performance by changing the average number of frame arrivals per beacon frame, denoted as $\overline{N_{a r r}}$. We introduce a new performance index, $G_{E_{\mathrm{b}}}$, to evaluate the relative gain of TRIPL over BASE in terms of energy efficiency; that is, $G_{E_{\mathrm{b}}}=\left(E_{\mathrm{b}}(\mathrm{BASE})-E_{\mathrm{b}}(\mathrm{TRIPL})\right) / E_{\mathrm{b}}(\mathrm{BASE})$. Figure 6 shows $G_{E_{\mathrm{b}}}$ obtained under Scenario 4 where the value of $\overline{N_{\text {arr }}}$ ranges between 1 and 10 according to a Poisson distribution, and $N_{\text {bg }}$ has different values of 4,6 , and 10 . As $\overline{N_{\text {arr }}}$ increases, $G_{E_{\mathrm{b}}}$ increases due to sufficient number of frames to aggregate and the increase of semisleep time. Recall that the proposed mechanism aims to improve energy efficiency during the active states by effectively dealing with the ILT, whereas there

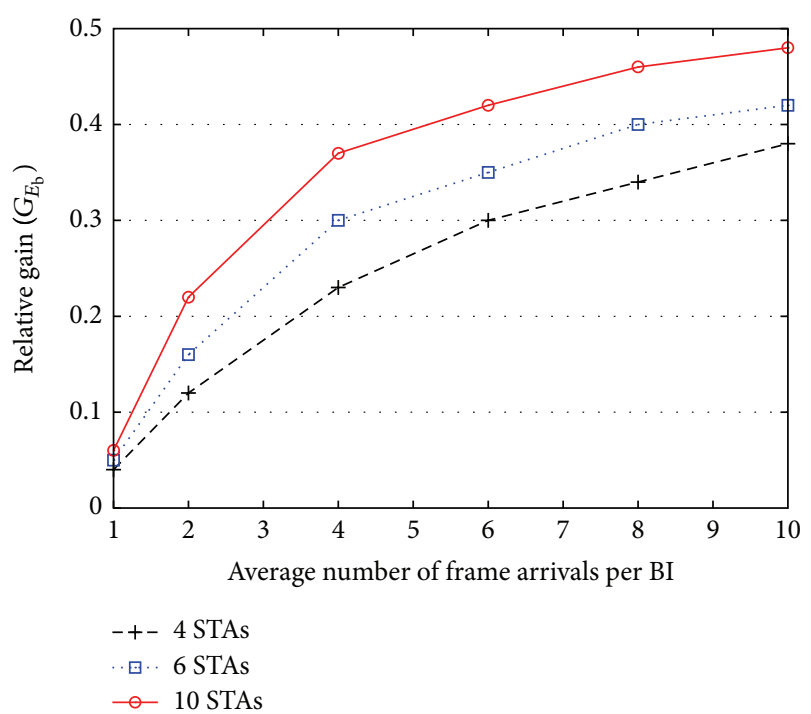

FIGURE 6: Relative gain of TRIPL over BASE in terms of $E_{\mathrm{b}}$ (Scenario 4).

is no way to improve it during the sleep states. In addition, TRIPL attains higher $G_{E_{\mathrm{b}}}$ when there are many background STAs, because its gain is boosted by effectively decreasing collision. When $N_{\mathrm{bg}}=10$ and $\overline{N_{\mathrm{arr}}}=10, G_{E_{\mathrm{b}}}$ achieves the maximum value of 0.48 . When $\overline{N_{\text {arr }}}=2, G_{E_{\mathrm{b}}}$ is about 0.13 , 0.15 , and 0.21 in the cases of $N_{\mathrm{bg}}=4,6$, and 10, respectively. The results in Figure 6 indicate that TRIPL further improves energy efficiency when the traffic load is high and the network is dense with many STAs, which is a clearly increasing trend in the current and future WLANs.

\section{Conclusion}

In this paper, we focused on the problem of energy loss due to IL in IEEE 802.11 WLANs and verified that the IL is the primary obstacle to improving energy efficiency of WLANs. We also identified three sources of IL: overhearing nonintended frames, channel sensing and accessing based on backoff, and collision among STAs. As a solution to this problem, we proposed the energy-efficient mechanism consisting of three schemes: downclocking, frame aggregation, and contention window control. Each of these schemes is designed to alleviate a cause of IL. Firstly, the downclocking scheme allows the STA overhearing nonintended frames to remain in the semisleep state. Secondly, the frame aggregation scheme extends the semisleep time, as well as decreasing the channel sensing and accessing overhead per frame. Lastly, the contention window control scheme decreases collisions and avoids unnecessary retransmissions. Therefore, the proposed mechanism not only significantly improves energy efficiency but also increases total throughput. By deriving the analysis model and comparing the analysis results with simulation results, we validated the outstanding performance of the proposed mechanism in terms of energy per bit and throughput. 


\section{Competing Interests}

The authors declare that they have no competing interests.

\section{Acknowledgments}

This work was supported by the National Research Foundation of Korea (NRF) grant funded by the Korean government (MSIP) (no. NRF-2013R1A2A2A01009883).

\section{References}

[1] G. Anastasi, M. Conti, E. Gregori, and A. Passarella, "802.11 power-saving mode for mobile computing in Wi-Fi hotspots: limitations, enhancements and open issues," Wireless Networks, vol. 14, no. 6, pp. 745-768, 2008.

[2] Y. Agarwal, R. Chandra, A. Wolman, P. Bahl, K. Chin, and R. Gupta, "Wireless wakeups revisited: energy management for VoIP over Wi-Fi smartphones," in Proceedings of the 5th International Conference on Mobile Systems, Applications and Services (MobiSys '07), pp. 179-191, June 2007.

[3] IEEE 802.11 Working Group, "Part 11: wireless LAN medium access control (MAC) and physical layer (PHY) specifications," IEEE Standard 802.11-2007, 2007.

[4] X. Zhang and K. G. Shin, "E-mili: energy-minimizing idle listening in wireless networks," IEEE Transactions on Mobile Computing, vol. 11, no. 9, pp. 1441-1454, 2012.

[5] D. Levy and I. Kotuliak, "WLAN power saving using packet overhearing reduction," Telecommunication Systems, vol. 61, no. 1, pp. 43-57, 2016.

[6] M. Adnan and E.-C. Park, "Hybrid control of contention window and frame aggregation for performance enhancement in multirate WLANs," Mobile Information Systems, vol. 2015, Article ID 383081, 16 pages, 2015.

[7] R. P. Liu, G. J. Sutton, and I. B. Collings, "WLAN power save with offset listen interval for machine-to-machine communications," IEEE Transactions on Wireless Communications, vol. 13, no. 5, pp. 2552-2562, 2014.

[8] Y.-H. Zhu, H.-C. Lu, and V. C. M. Leung, "Access point buffer management for power saving in IEEE 802.11 WLANs," IEEE Transactions on Network and Service Management, vol. 9, no. 4, pp. 473-486, 2012.

[9] S.-L. Tsao and C.-H. Huang, "A survey of energy efficient MAC protocols for IEEE 802.11 WLAN," Computer Communications, vol. 34, no. 1, pp. 54-67, 2011.

[10] IEEE 802.11 Working Group, "Part 11: wireless LAN medium access control (MAC) and physical layer (PHY) specifications. amendment 8: medium access control (MAC) quality of service enhancements," IEEE Standard 802.11e-2005, 2005.

[11] D. C. Mur, X. Pérez-Costa, and S. S. Ribes, "An adaptive solution for wireless LAN distributed power saving modes," Computer Networks, vol. 53, no. 18, pp. 3011-3030, 2009.

[12] IEEE 802.11 Working Group, "Part 11: Wireless LAN medium access control (MAC) and physical layer (PHY) specifications. amendment 5: enhancements for higher throughput," IEEE Std 802.11n-2009, 2009.

[13] H. Singh, H.-R. Shao, and C. Ngo, "Enhanced power saving in next generation wireless LANs," in Proceedings of IEEE Vehicular Technology Conference (VTC)-Fall, pp. 1-5, Montreal, Canada, September 2006.
[14] F. M. Abinader, E. P. L. Almeida, S. Choudhury et al., "Performance evaluation of IEEE 802.11n WLAN in dense deployment scenario," in Proceedings of the 80th IEEE Vehicular Technology Conference (VTC '14-Fall), Vancouver, Canada, September 2014.

[15] S. Biswas and S. Datta, "Reducing overhearing energy in 802.11 Networks by low-power interface idling," in Proceedings of IEEE International Conference on Performance, Computing, and Communications (IPCCC '04), pp. 695-700, April 2004.

[16] B. Balaji, B. R. Tamma, and B. S. Manoj, "A novel power saving strategy for greening IEEE 802.11 based wireless networks," in Proceedings of the 53rd IEEE Global Communications Conference (GLOBECOM '10), pp. 1-5, Miami, Fla, USA, December 2010.

[17] V. Baiamonte and C.-F. Chiasserini, "Saving energy during channel contention in 802.11 WLANs," Mobile Networks and Applications, vol. 11, no. 2, pp. 287-296, 2006.

[18] J. Liu and L. Zhong, "Micro power management of active 802.11 interfaces," in Proceedings of the ACM International Conference on Mobile Systems, Applications, and Services (MobiSys '08), pp. 146-159, Breckenridge, Colo, USA, June 2008.

[19] B. Tavli and W. B. Heinzelman, "MH-TRACE: multihop time reservation using adaptive control for energy efficiency," IEEE Journal on Selected Areas in Communications, vol. 22, no. 5, pp. 942-953, 2004.

[20] C. Suh, Y.-B. Ko, and J.-H. Kim, "Enhanced power saving for IEEE 802.11 WLAN with dynamic slot allocation," in Mobile Adhoc and Sensor Networks, vol. 3794 of Lecture Notes in Computer Science, pp. 498-507, 2005.

[21] Y. He and R. Yuan, "A novel scheduled power saving mechanism for 802.11 wireless LANs," IEEE Transactions on Mobile Computing, vol. 8, no. 10, pp. 1368-1383, 2009.

[22] X. Lei and S. H. Rhee, "Enhancement of the IEEE 802.11 power saving mode by prioritized reservations," International Journal of Distributed Sensor Networks, vol. 2015, Article ID 586289, 10 pages, 2015.

[23] K. Flautner, S. Reinhardt, and T. Mudge, "Automatic performance setting for dynamic voltage scaling," in Proceedings of the 7th Annual International Conference on Mobile Computing and Networking (MobiCom '01), pp. 260-271, July 2001.

[24] T. Li, Q. Ni, D. Malone, D. Leith, Y. Xiao, and T. Turletti, "Aggregation with fragment retransmission for very high-speed WLANs," IEEE/ACM Transactions on Networking, vol. 17, no. 2, pp. 591-604, 2009.

[25] G. Bianchi, "Performance analysis of the IEEE 802.11 distributed coordination function," IEEE Journal on Selected Areas in Communications, vol. 18, no. 3, pp. 535-547, 2000.

[26] F. Cali, M. Conti, and E. Gregori, "Dynamic tuning of the IEEE 802.11 protocol to achieve a theoretical throughput limit," IEEE/ACM Transactions on Networking, vol. 8, no. 6, pp. 785799, 2000.

[27] D.-Y. Yang, T.-J. Lee, K. Jang, J.-B. Chang, and S. Choi, "Performance enhancement of multirate IEEE 802.11 WLANs with geographically scattered stations," IEEE Transactions on Mobile Computing, vol. 5, no. 7, pp. 906-919, 2006.

[28] B. Li, R. Battiti, and Y. Fang, "Achieving optimal performance by using the IEEE 802.11 MAC protocol with service differentiation enhancements," IEEE Transactions on Vehicular Technology, vol. 56, no. 3, pp. 1374-1387, 2007.

[29] A. V. Babu and L. Jacob, "Fairness analysis of IEEE 802.11 multirate wireless LANs," IEEE Transactions on Vehicular Technology, vol. 56, no. 5, pp. 3073-3088, 2007. 
[30] L. M. Feeney and M. Nilsson, "Investigating the energy consumption of a wireless network interface in an ad hoc networking environment," in Proceedings of the IEEE 20th Annual Joint Conference of the IEEE Computer and Communications Societies (INFOCOM '01), vol. 3, pp. 1548-1557, IEEE, Anchorage, Alaska, USA, April 2001.

[31] P. Serrano, A. Garcia-Saavedra, G. Bianchi, A. Banchs, and A. Azcorra, "Per-frame energy consumption in 802.11 devices and its implication on modeling and design," IEEE/ACM Transactions on Networking, vol. 23, no. 4, pp. 1243-1256, 2015. 

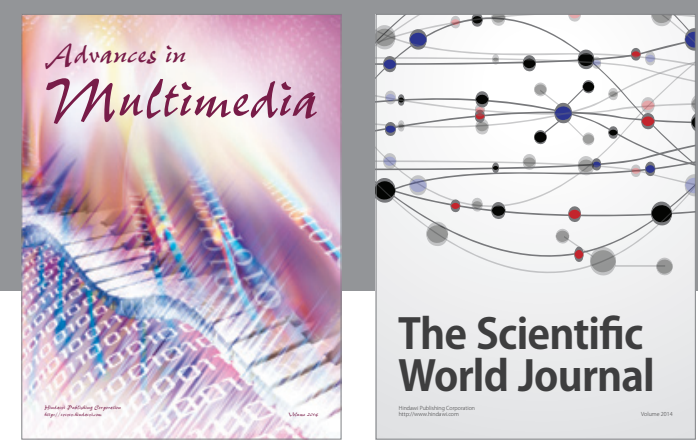

The Scientific World Journal
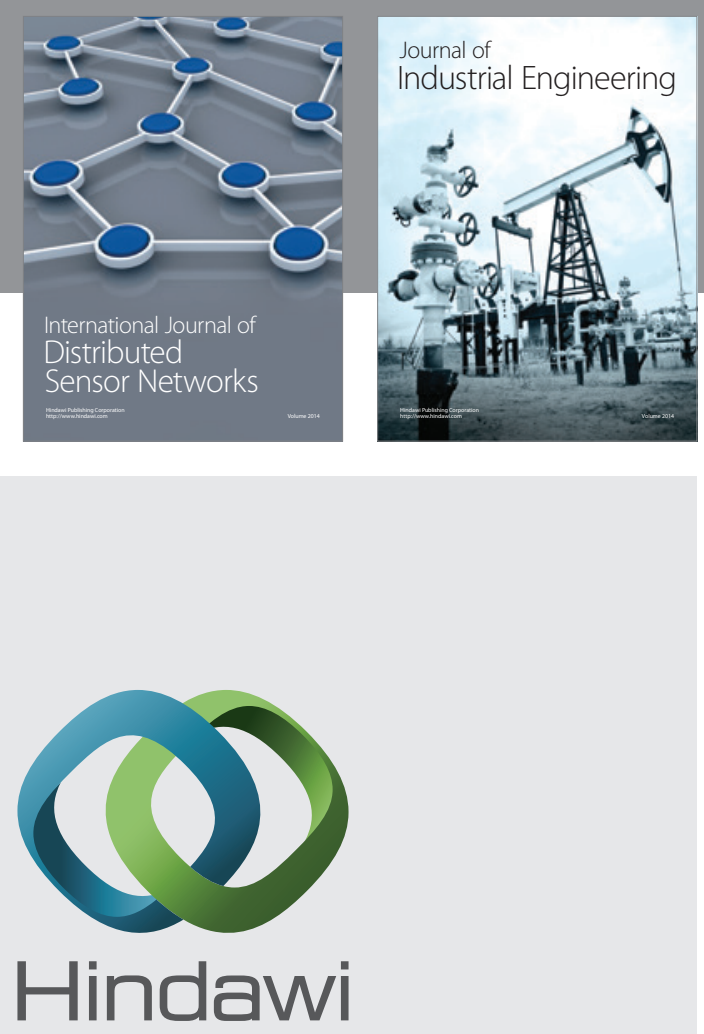

Submit your manuscripts at

http://www.hindawi.com

\section{Computer Networks} and Communications
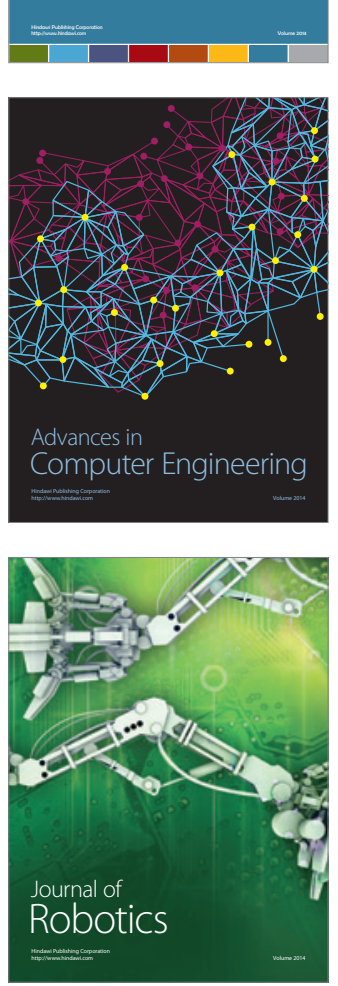
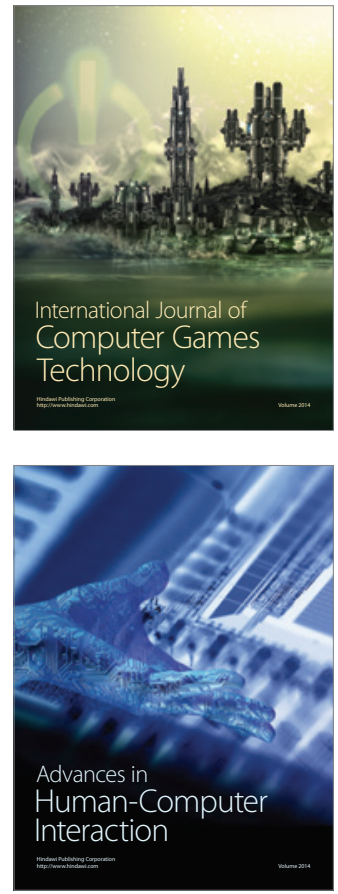
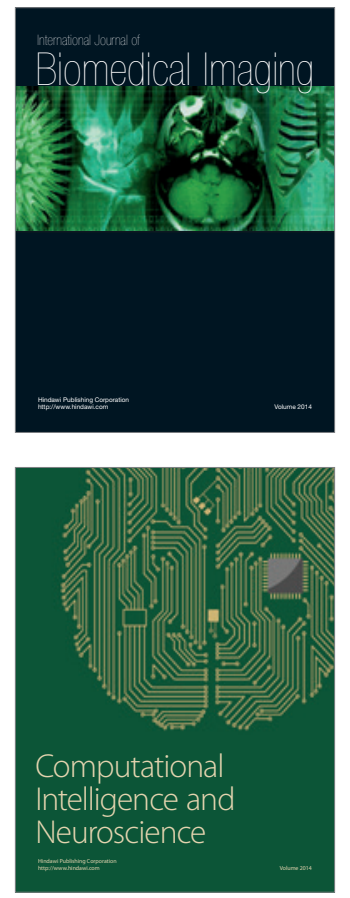
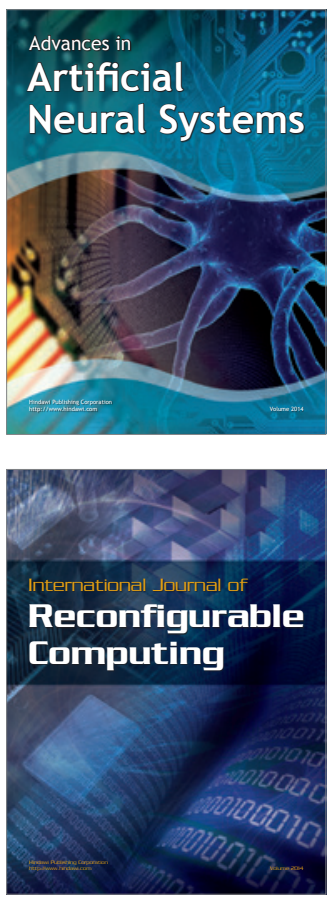
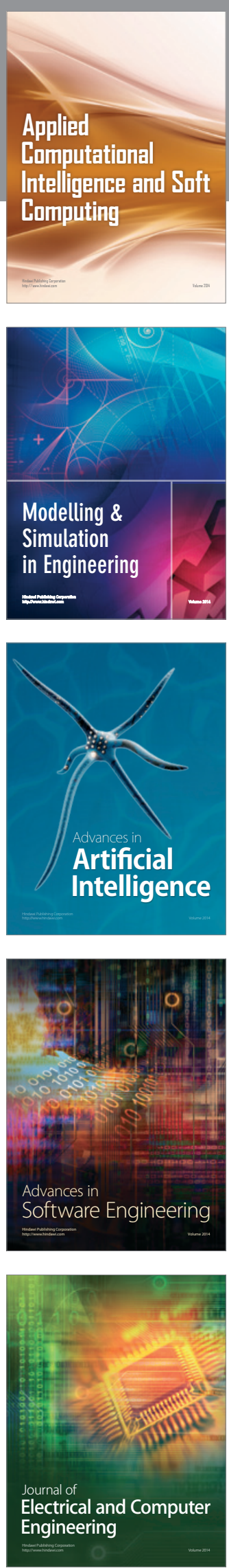